ULM-TP/01-04

HPL-BRIMS-2001-11

April 2001

\title{
Autocorrelation function of eigenstates in chaotic and mixed systems
}

\author{
Arnd Bäcker円² and Roman Schubert円 \\ 1) School of Mathematics, University of Bristol \\ University Walk, Bristol BS8 1TW, UK \\ 2) BRIMS, Hewlett-Packard Laboratories \\ Filton Road, Bristol BS12 6QZ, UK \\ 3) Abteilung Theoretische Physik, Universität Ulm, \\ Albert-Einstein-Allee 11, D-89069 Ulm, Germany
}

\begin{abstract}
:
We study the autocorrelation function of different types of eigenfunctions in quantum mechanical systems with either chaotic or mixed classical limits. We obtain an expansion of the autocorrelation function in terms of the correlation length. For localized states, like bouncing ball modes or states living on tori, a simple model using only classical input gives good agreement with the exact result. In particular, a prediction for irregular eigenfunctions in mixed systems is derived and tested. For chaotic systems, the expansion of the autocorrelation function can be used to test quantum ergodicity on different length scales.
\end{abstract}

\footnotetext{
${ }^{1}$ E-mail address: a.backer@bristol.ac.uk

${ }^{3}$ E-mail address: roman.schubert@physik.uni-ulm.de
} 


\section{Introduction}

The behavior of a quantum mechanical system in the semiclassical limit strongly depends on the ergodic properties of the corresponding classical system. In particular, the eigenfunctions semiclassically reflect the phase space structure of the classical system and therefore they depend strongly on whether the classical system is chaotic or regular. In this work we are interested in the fluctuations of the wavefunctions, and in the correlations between the fluctuations in different regions which are induced by the classical phase space structures. In particular, we will consider the case of quantum billiards in a domain $\Omega \subset \mathbb{R}^{2}$, which are described by the time independent Schrödinger equation

$$
(\Delta+E) \psi(\mathbf{q})=0 \quad \text { for } \mathbf{q} \in \Omega \backslash \partial \Omega,
$$

with Dirichlet boundary conditions, $\psi(\mathbf{q})=0$ for $\mathbf{q} \in \partial \Omega$. For compact $\Omega$ one obtains a discrete spectrum $\left\{E_{n}\right\}$ of eigenvalues, $0<E_{1} \leq E_{2} \leq \ldots$, with associated eigenfunctions $\psi_{n} \in L^{2}(\Omega)$,

which we assume to be normalized, i.e. ||$\psi_{n}||:=\int_{\Omega}\left|\psi_{n}(\mathbf{q})\right|^{2} \mathrm{~d} q=1$. The corresponding classical billiard is given by the free motion of a point particle inside $\Omega$ with elastic reflections at the boundary $\partial \Omega$.

The amplitude distribution of an eigenfunction of a quantum mechanical system whose classical limit is chaotic is conjectured to become Gaussian in the semiclassical limit [1], and numerical studies support this conjecture, see e.g. 2 2 4]. A more sensitive quantity is the local autocorrelation function [1] which measures correlations between different points of an eigenfunction $\psi$,

$$
C^{\text {loc }}(\mathbf{x}, \delta \mathbf{x}):=\psi^{*}(\mathbf{x}-\delta \mathbf{x} / 2) \psi(\mathbf{x}+\delta \mathbf{x} / 2)
$$

The crucial fact for the theoretical analysis of $C^{\text {loc }}(\mathbf{x}, \delta \mathbf{x})$, observed by Berry [1], is that the autocorrelation function can be expressed as the Fourier transformation of the Wigner function (see eq. (7) below) of $\psi$,

$$
C^{\mathrm{loc}}(\mathbf{x}, \delta \mathbf{x})=\int W(\mathbf{p}, \mathbf{x}) \mathrm{e}^{-\mathrm{i} \mathbf{p} \delta \mathbf{x}} \mathrm{d} p
$$

Hence information on the behavior of the Wigner function can be used to predict the behavior of the autocorrelation function, and since semiclassical limits of Wigner functions are concentrated on invariant sets in phase space, see e.g. [5], it follows that in the semiclassical limit autocorrelation functions are determined by the classical phase space structure. For example, if the classical system is ergodic, the quantum ergodicity theorem [6-11] (roughly speaking) states that almost all quantum expectation values tend to the corresponding classical limit. One can show [12] that for ergodic systems this is equivalent to the semiclassical eigenfunction hypothesis [13, 1, 14, 15], when restricted to a subsequence of density one. Using this result in (3) one gets Berry's result [1] that for chaotic billiards in two dimensions

$$
C^{\mathrm{loc}}(\mathbf{x}, \delta \mathbf{x}) \sim \frac{1}{\operatorname{vol}(\Omega)} J_{0}(\sqrt{E}|\delta \mathbf{x}|)
$$

weakly as a function of $\mathbf{x}$ (for fixed $\delta \mathbf{x}$ ) as $E \rightarrow \infty$, where $E$ denotes the energy of the eigenstate $\psi$ in (2). Equivalently we have

$$
\lim _{E \rightarrow \infty} C^{\mathrm{loc}}(\mathbf{x}, \delta \mathbf{x} / \sqrt{E})=\frac{1}{\operatorname{vol}(\Omega)} J_{0}(|\delta \mathbf{x}|) .
$$


Numerical and experimental tests of this relation have been performed for several chaotic systems [2 [4, 16] and show at finite energies noticeable fluctuations of the autocorrelation function around the high energy limit (田), especially for correlation distances larger than a few de Broglie wavelengths. These fluctuations have been studied further in [17 [20], where for small correlation length $|\delta \mathbf{x}|$ a random model for the eigenfunctions of a chaotic system was used to predict the variance of these fluctuations, and for larger $|\delta \mathbf{x}|$ a formula involving closed orbits of the system has been derived. In [21, 22] the path correlation function, which is an average of the local correlations along a given trajectory, has been introduced. The path correlation function is closely related to the autocorrelation function and for ergodic systems also tends asymptotically to a Bessel function (4). This path correlation function has been studied in [3] for an hyperbolic octagon, and an expansion in terms of Legendre functions has been derived, which can be used to determine corrections to the leading Bessel part (四).

The autocorrelation function in non-chaotic systems has attracted much less attention. The integrable case has already been discussed by Berry [1], and the corresponding formula has been successfully tested for the circle billiard in [2]. For a system with mixed classical phase space the autocorrelation function has been studied in [23], in particular for irregular eigenfunctions an expansion of the Wigner function in polar coordinates has been used.

In this work we are interested in the question how the universal limit (田) is reached, and how, in the case of mixed systems, further constraints on the classical motion are reflected in the autocorrelation function. For instance, if an eigenfunction is concentrated on an ergodic component, then by a generalization of the quantum ergodicity theorem [24], the Wigner function becomes equidistributed on that component, and this will determine the autocorrelation function.

The paper is organized as follows. In section 2 we discuss some examples of the autocorrelation function for different eigenfunctions in systems with chaotic and mixed classical dynamics. In section 3 a general expansion of the autocorrelation function for eigenfunctions in billiards is derived, which allows a systematic study of their properties. It is an expansion in the correlation length $|\delta \mathbf{x}|$ which reflects the fact that the determination of correlations at larger distances needs classical information on finer length scales than for short range correlations. In section tit is shown that the correlation length expansion provides an efficient way to explain the fine structure of the autocorrelation functions of the systems studied in the first section. Of particular interest is that for chaotic systems deviations of the autocorrelation function from the quantum ergodic limit (4) can be related to the rate of quantum ergodicity. In turn the autocorrelation function can be used to study the rate of quantum ergodicity on different classical length scales.

\section{Examples of autocorrelation functions}

For numerical computations as well as for theoretical considerations it is much more convenient to consider a smoothed version of the local autocorrelation function (2). Furthermore, as the eigenfunctions oscillate on a scale proportional to $1 / \sqrt{E}$, we rescale the autocorrelation function by this factor. Hence we will study the autocorrelation function in the form

$$
C_{\rho}(\mathbf{x}, \delta \mathbf{x}):=\int_{\Omega} \rho(\mathbf{x}-\mathbf{q}) \psi^{*}\left(\mathbf{q}-\frac{\delta \mathbf{x}}{2 \sqrt{E}}\right) \psi\left(\mathbf{q}+\frac{\delta \mathbf{x}}{2 \sqrt{E}}\right) \mathrm{d} q,
$$

where $\rho$ is a positive function which determines the smoothing of the local autocorrelation function. In the literature (see the papers mentioned in the introduction) the mean is usually 
taken over a small disk, which corresponds to taking the characteristic function of a disk for $\rho$ in (6). However, nothing prevents one to consider the case $\rho \equiv 1$, i.e. to take the mean value of the local autocorrelation function (2) over the whole position space. In terms of the Wigner function

$$
W(\mathbf{p}, \mathbf{q}):=\frac{1}{(2 \pi)^{2}} \int \mathrm{e}^{\mathrm{i} \mathbf{p q}^{\prime}} \psi^{*}\left(\mathbf{q}-\mathbf{q}^{\prime} / 2\right) \psi\left(\mathbf{q}+\mathbf{q}^{\prime} / 2\right) \mathrm{d} q^{\prime}
$$

one obtains in this case

$$
\begin{aligned}
C(\delta \mathbf{x}) & :=\int \psi^{*}\left(\mathbf{q}-\frac{\delta \mathbf{x}}{2 \sqrt{E}}\right) \psi\left(\mathbf{q}+\frac{\delta \mathbf{x}}{2 \sqrt{E}}\right) \mathrm{d} q \\
& =\iint W(\mathbf{p}, \mathbf{q}) \mathrm{e}^{-\mathrm{i} \mathbf{p} \delta \mathbf{x} / \sqrt{E}} \mathrm{~d} q \mathrm{~d} p=\int|\widehat{\psi}(\mathbf{p})|^{2} \mathrm{e}^{-\mathrm{i} \mathbf{p} \delta \mathbf{x} / \sqrt{E}} \mathrm{~d} p
\end{aligned}
$$

This is a particularly good choice for the numerical computation of the autocorrelation function in billiards because it can be reduced to boundary integrals, see Appendix A. The resulting formula reads

$$
C(\delta \mathbf{x})=\frac{1}{8 \sqrt{E}} \iint_{\partial \Omega \times \partial \Omega}\left|\mathbf{q}(s)-\mathbf{q}\left(s^{\prime}\right)+\delta \mathbf{x}\right| Y_{1}\left(\sqrt{E}\left|\mathbf{q}(s)-\mathbf{q}\left(s^{\prime}\right)+\delta \mathbf{x}\right|\right) u^{*}(s) u\left(s^{\prime}\right) \mathrm{d} s \mathrm{~d} s^{\prime}
$$

where $u(s)$ is the normal derivative of the normalized eigenfunction $\psi$ on the billiard boundary. This relation provides a very efficient method for the numerical computation of the autocorrelation function.

The systems for which we study the autocorrelation functions are the stadium billiard and two members of the family of limaçon billiards, namely the cardioid billiard, and a billiard with mixed classical phase space. The stadium billiard is proven to be strongly chaotic, i.e. it is ergodic, mixing and a $K$-system [25,26]. The height of the desymmetrized billiard is chosen to be 1 , and $a$ denotes the length of the upper horizontal line, for which we have $a=1.8$ in the following. The family of limaçon billiards is given by the simplest nontrivial conformal mapping of the unit circle [27,28] and can be parametrized in polar coordinates by $\rho(\varphi)=1+\varepsilon \cos (\varphi)$ with $\varphi \in[-\pi, \pi]$ and $\varepsilon \in[0,1]$ denotes the family parameter. We consider the case $\varepsilon=0.3$ which leads to a mixed dynamics in phase space. For $\varepsilon=1$ one obtains the cardioid billiard, which is also proven to be strongly chaotic [29 31]. The eigenvalues of the cardioid billiard have been provided by Prosen and Robnik [32 and were calculated by means of the conformal mapping technique, see e.g. [28, 33]. For the stadium billiard the eigenvalues and eigenfunctions have been computed using the boundary element method, see e.g. [34, 35], and for the limaçon billiard the eigenvalues have been computed using the conformal mapping technique and then the boundary element method has been used to compute the eigenfunctions (see [36] for details). For the high lying states in the limaçon billiard the scaling method has been used [37].

First we consider a "typical" eigenfunction in the cardioid billiard, see fig. 11. In the plots we show

$$
C(r, \theta)=C(r \hat{\mathbf{e}}(\theta))
$$

where $\hat{\mathbf{e}}(\theta)=(\cos \theta, \sin \theta)$, as a function of $r$ for three different values of $\theta$. The quantum ergodicity theorem implies that there is a subsequence $\left\{n_{j}\right\} \subset \mathbb{N}$ of density one such that $C_{n_{j}}(r, \theta) \rightarrow J_{0}(r)$ as $n_{j} \rightarrow \infty$ with $r$ fixed. This convergence is, however, not uniform in $r$. 

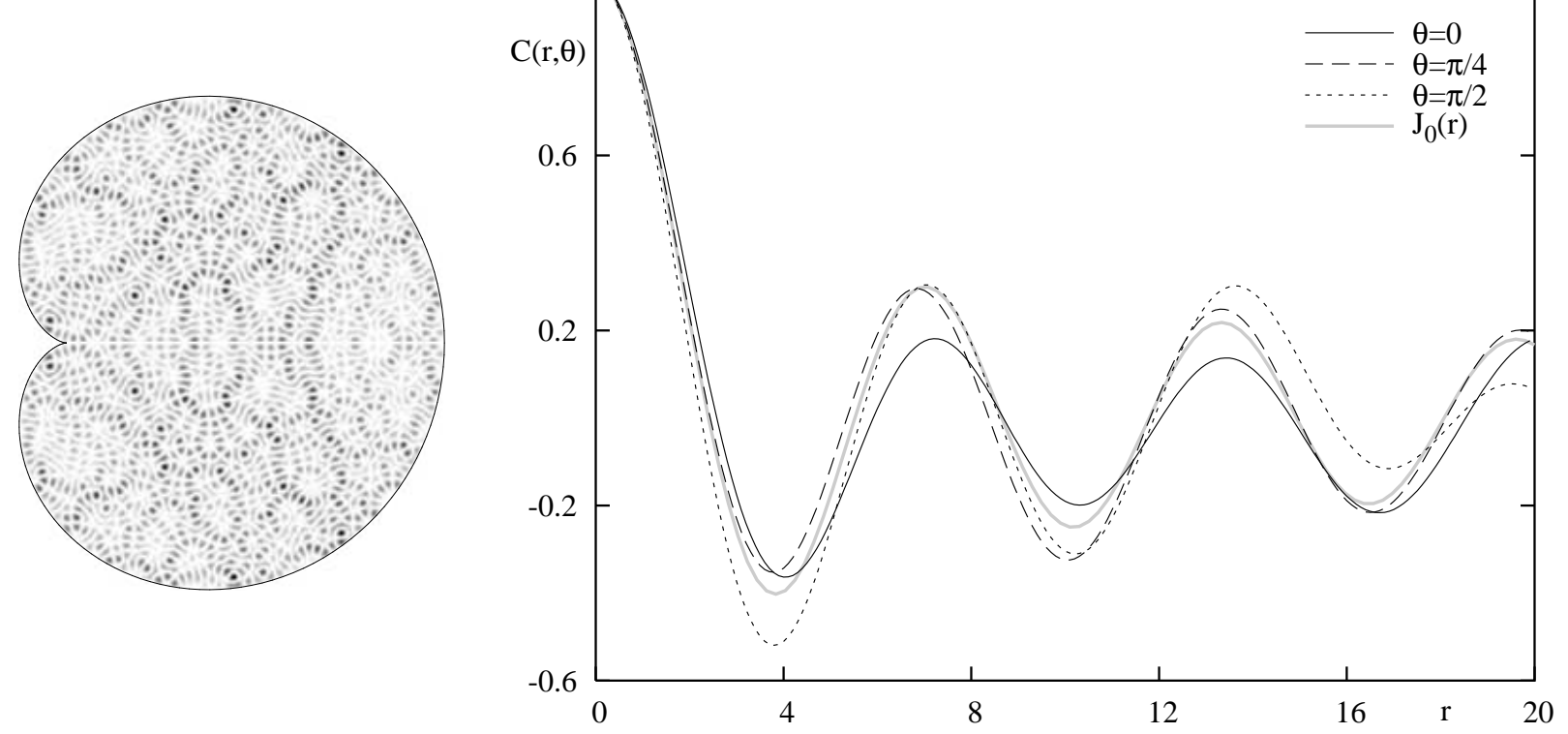

Figure 1: Grey scale plot of $\left|\psi_{1277}(\mathbf{q})\right|^{2}$ in the cardioid billiard with odd symmetry, where black corresponds to high intensity. To the right the autocorrelation function $C(r, \theta)$, computed using (10), is shown for three different directions $\theta=0, \pi / 4$ and $\pi / 2$. For comparison the asymptotic result $C(r, \theta)=J_{0}(r)$ is shown as grey line.

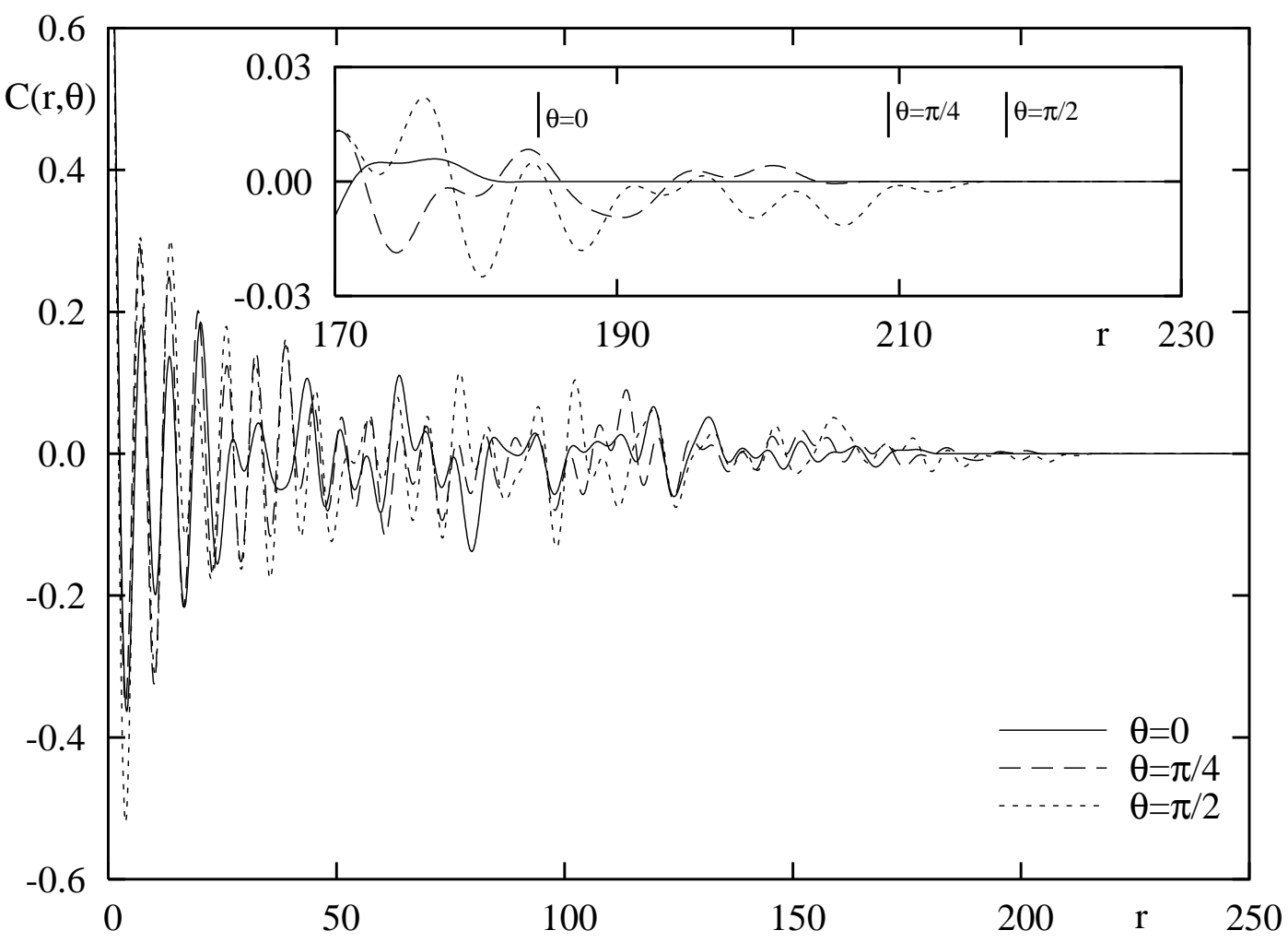

Figure 2: Autocorrelation function for the same state as fig. [, but for a larger $r$-interval showing the non-universal behavior at larger $r$. The inset shows a magnification and the vertical bars indicate the places $r=\sqrt{E_{n}} \operatorname{diam}(\Omega, \theta)$ from where on $C(r, \theta)=0$, due to the compactness of the billiard. 
For the example shown in fig. 1 $C(r, \theta)$ fluctuates, as expected for a "quantum ergodic" state, around the asymptotic result

$$
C(r, \theta) \sim J_{0}(r)
$$

Actually, for an eigenstate with energy $E_{n}$ we have $C(r, \theta)=0$ for $r>\sqrt{E_{n}} \operatorname{diam}(\Omega, \theta)$, where $\operatorname{diam}(\Omega, \theta)$ is the diameter of $\Omega$ in the direction $\theta$, as follows directly from the definition (6). This is illustrated in fig. 2 which clearly shows the non-universal behavior for larger $r$.

In contrast to the case of quite uniformly distributed eigenfunctions one expects a stronger directional dependence of the autocorrelation function for localized eigenfunctions, such as scars [38]. One example is shown in fig. 3, where the eigenfunction shows localization along the shortest unstable periodic orbit in the cardioid. The corresponding autocorrelation function shows clear deviations from (12).

A class of eigenfunctions which show even stronger localization are the bouncing ball modes in billiards with two parallel walls, see e.g. [39,2, 40,42]. Fig. 14 shows for the stadium billiard an example of a bouncing ball mode, which localize on the so-called bouncing ball orbits having perpendicular reflections at the parallel walls and thus forming a one-parameter family. The simplest approximation is to consider them as a product of two sines, one in the $x$ direction and the other in the $y$ direction. In this case the autocorrelation function can be computed explicitly. For the odd-odd eigenfunctions

$$
\psi_{n_{x}, n_{y}}(x, y)=\frac{1}{\sqrt{l_{x} l_{y}}} \sin \left(\pi n_{x} x / l_{x}\right) \sin \left(\pi n_{y} y / l_{y}\right)
$$

in a box $B:=\left[-l_{x}, l_{x}\right] \times\left[-l_{y}, l_{y}\right]$ one gets

$$
C_{n_{x}, n_{y}}^{\text {box }}(r, \theta)=F\left(r \cos (\theta) / \sqrt{E}, n_{x}, l_{x}\right) F\left(r \sin (\theta) / \sqrt{E}, n_{y}, l_{y}\right)
$$
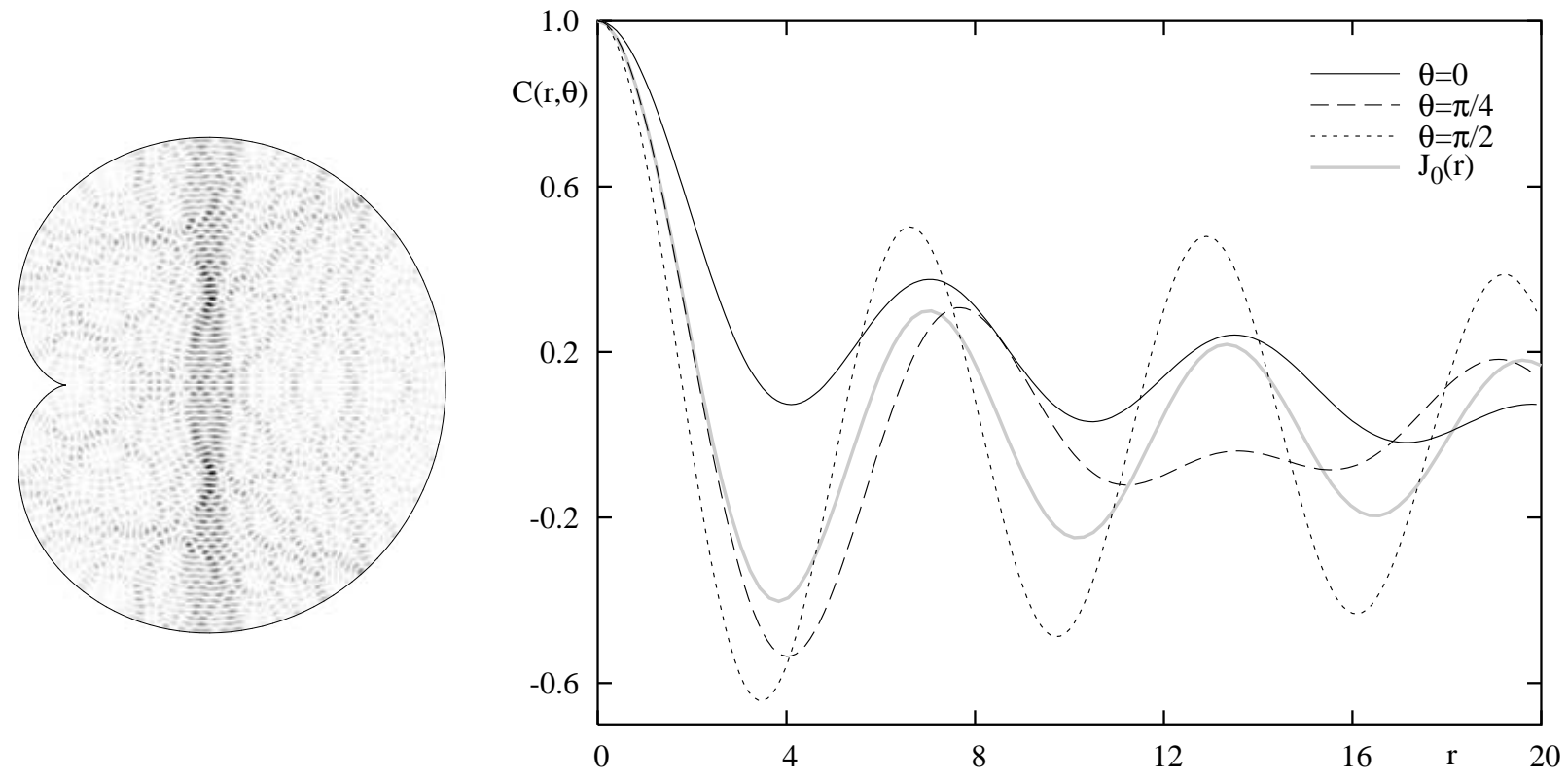

Figure 3: Grey scale plot of $\left|\psi_{n}(\mathbf{q})\right|^{2}$ with $n=1817$ in the cardioid billiard with odd symmetry. For the autocorrelation function $C(r, \theta)$ one observes clear deviations from $C(r, \theta)=J_{0}(r)$. 

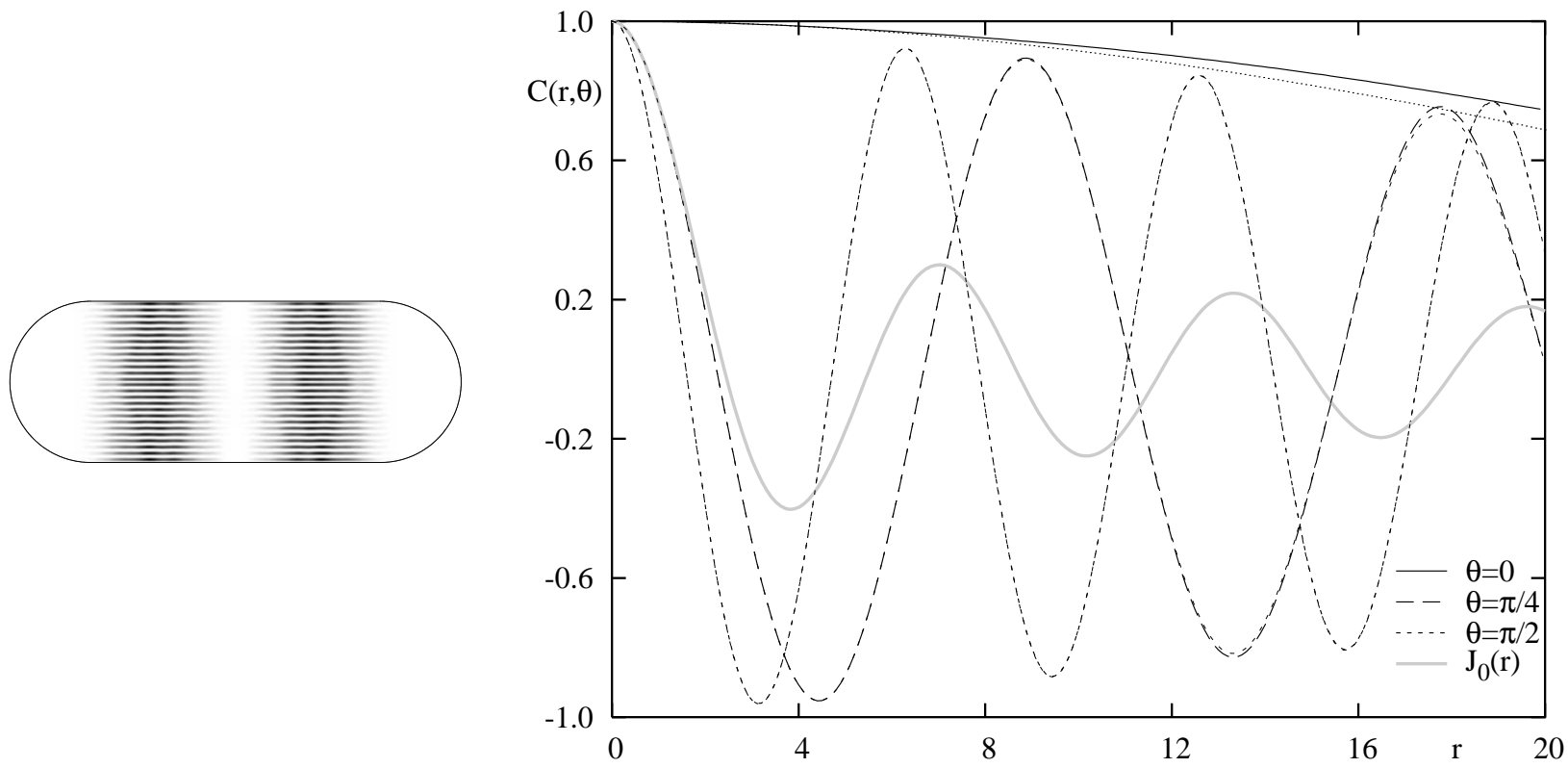

Figure 4: For the stadium billiard with odd-odd symmetry, $a=1.8, \psi_{320}(\mathbf{q})$ is a bouncing ball mode. The corresponding autocorrelation function is compared with the result $C_{1,13}^{\text {box }}(r, \theta)$, eq. (14), obtained for a box, shown as dotted curves, which follow $C(r, \theta)$. Only for $\theta=0$ (full line) and $\theta=\pi / 4$ at $r \approx 17$ are small deviations visible.

where

$$
\begin{aligned}
F(z, n, l) & :=\chi_{[-l, l]}(z / 2) \frac{1}{l} \int_{-l+z / 2}^{l-z / 2} \sin (\pi n(x-z / 2) / l) \sin (\pi n(x+z / 2) / l) \mathrm{d} x \\
& =\chi_{[-l, l]}(z / 2)\left[\left(1-\frac{z}{2 l}\right) \cos (\pi n z / l)+\frac{1}{2 \pi n} \sin (\pi n z / l)\right],
\end{aligned}
$$

and $\chi_{[-l, l]}(z)$ denotes the characteristic function of the interval $[-l, l]$.

In fig. 4 we compare the autocorrelation function $C(r, \theta)$ for a bouncing ball mode in the stadium billiard with $C_{1,13}^{\text {box }}(r, \theta)$, eq. (14), and observe very good agreement. Mainly for $\theta=0$ some deviations are visible; these are understandable from the fact that in this case only correlations in the $x$-direction are measured, where the bouncing ball mode "leaks" outside the rectangular region. To take this into account one can determine an effective $l_{x}^{\text {eff }}>2 a$, by fitting $\sin ^{2}\left(\pi x / l_{x}^{\text {eff }}\right)$ to

$$
\psi_{n}^{\operatorname{proj}}(x):=\int_{0}^{1}|\psi(x, y)|^{2} \mathrm{~d} y
$$

For the case shown in fig. 1 this procedure leads to $l_{x}^{\text {eff }} \approx 4$ (whereas $2 a=3.6$ ) and the corresponding autocorrelation function gives excellent agreement with the one for $\psi_{320}$. 


\section{Expansion of the autocorrelation function}

In this section we derive an expansion of the autocorrelation function which will lead to an understanding of the directional dependence of the autocorrelation function observed in the last section. We start from the representation of the local autocorrelation function in terms of the Wigner function

$$
C_{\rho}(\mathbf{x}, \delta \mathbf{x})=\iint \rho(\mathbf{x}-\mathbf{q}) W(\mathbf{p}, \mathbf{q}) \mathrm{e}^{-\mathrm{i} p \delta \mathbf{x} / \sqrt{E}} \mathrm{~d} p \mathrm{~d} q
$$

Since the Wigner function is concentrated around the energy shell $|\mathbf{p}|=\sqrt{E}$, and is furthermore even in $\mathbf{p}$ by time reversal symmetry, we get

$$
\begin{aligned}
C_{\rho}(\mathbf{x}, \delta \mathbf{x}) & =\int_{0}^{\infty} \int_{0}^{2 \pi} \int_{\Omega} \rho(\mathbf{x}-\mathbf{q}) W(\mathbf{p}, \mathbf{q}) \mathrm{d} q \mathrm{e}^{-\mathrm{i}|\delta \mathbf{x}| \cos (\varphi-\theta)} r \mathrm{~d} \varphi \mathrm{d} r+O\left(E^{-1 / 2}\right) \\
& =\int_{0}^{\infty} \int_{0}^{2 \pi} \int_{\Omega} \rho(\mathbf{x}-\mathbf{q}) W(\mathbf{p}, \mathbf{q}) \mathrm{d} q \cos (|\delta \mathbf{x}| \cos (\varphi-\theta)) r \mathrm{~d} \varphi \mathrm{d} r+O\left(E^{-1 / 2}\right)
\end{aligned}
$$

where we have used polar coordinates $\mathbf{p}=(|\mathbf{p}| \cos \varphi,|\mathbf{p}| \sin \varphi), \delta \mathbf{x}=(|\delta \mathbf{x}| \cos \theta,|\delta \mathbf{x}| \sin \theta)$. Because of the rescaling by $\sqrt{E}$ the factor $\mathrm{e}^{-\mathrm{i} \mathbf{p} \delta \mathbf{x} / \sqrt{E}}$ is only slowly oscillating for $\mathbf{p}$ close to the energy shell, on which the Wigner function is concentrated. Therefore we get that the error is of order $1 / \sqrt{E}$, see Appendix B for a sketch of the derivation of this remainder estimate. If we now use that $\cos (r \cos \varphi)$ is a generating function for Bessel functions [43],

$$
\cos (|\delta \mathbf{x}| \cos \varphi)=J_{0}(|\delta \mathbf{x}|)+2 \sum_{l=1}^{\infty}(-1)^{l} \cos (2 l \varphi) J_{2 l}(|\delta \mathbf{x}|)
$$

we obtain

$$
C_{\rho}(\mathbf{x}, \delta \mathbf{x})=\xi_{0}(\mathbf{x}) J_{0}(|\delta \mathbf{x}|)+2 \sum_{l=1}^{\infty}(-1)^{l} \xi_{2 l}(\mathbf{x}, \theta) J_{2 l}(|\delta \mathbf{x}|)+O\left(E^{-1 / 2}\right)
$$

with (setting $r=|\mathbf{p}|$ )

$$
\xi_{2 l}(\mathbf{x}, \theta):=\int_{0}^{\infty} \int_{0}^{2 \pi} \int_{\Omega} \rho(\mathbf{x}-\mathbf{q}) W(\mathbf{p}, \mathbf{q}) \mathrm{d} q \cos (2 l(\varphi-\theta)) r \mathrm{~d} \varphi \mathrm{d} r .
$$

The coefficients $\xi_{2 l}(\mathbf{x}, \theta)$ can be further decomposed

$$
\begin{aligned}
\xi_{2 l}(\mathbf{x}, \theta)= & \cos (2 l \theta) \int_{0}^{\infty} \int_{0}^{2 \pi} \int_{\Omega} \rho(\mathbf{x}-\mathbf{q}) W(\mathbf{p}, \mathbf{q}) \mathrm{d} q \cos (2 l \varphi) r \mathrm{~d} \varphi \mathrm{d} r \\
& +\sin (2 l \theta) \int_{0}^{\infty} \int_{0}^{2 \pi} \int_{\Omega} \rho(\mathbf{x}-\mathbf{q}) W(\mathbf{p}, \mathbf{q}) \mathrm{d} q \sin (2 l \varphi) r \mathrm{~d} \varphi \mathrm{d} r
\end{aligned}
$$


Recall that for an operator $\widehat{A}$ with Weyl symbol $A(\mathbf{p}, \mathbf{q})$ the expectation value $\langle\psi, \widehat{A} \psi\rangle$ can be written as an integral over phase space of the symbol multiplied by the Wigner function of $\psi$, see e.g. [44,

$$
\langle\psi, \widehat{A} \psi\rangle=\iint W(\mathbf{p}, \mathbf{q}) A(\mathbf{p}, \mathbf{q}) \mathrm{d} p \mathrm{~d} q
$$

Therefore the coefficients in (23) can be interpreted as expectation values of certain operators $\widehat{A}_{2 l}(\mathbf{x}), \widehat{B}_{2 l}(\mathbf{x})$ given as the Weyl quantizations of the functions

$$
A_{2 l}(\mathbf{p}, \mathbf{q}):=\rho(\mathbf{x}-\mathbf{q}) \cos (2 l \varphi) \quad B_{2 l}(\mathbf{p}, \mathbf{q})=\rho(\mathbf{x}-\mathbf{q}) \sin (2 l \varphi)
$$

respectively,

$$
\begin{aligned}
& \int_{\Omega} \int_{0}^{2 \pi} \int_{0}^{\infty} W(\mathbf{p}, \mathbf{q}) \rho(\mathbf{x}-\mathbf{q}) \cos (2 l \varphi) r \mathrm{~d} r \mathrm{~d} \varphi \mathrm{d} q=\left\langle\psi, \widehat{A}_{2 l}(\mathbf{x}) \psi\right\rangle \\
& \int_{\Omega} \int_{0}^{2 \pi} \int_{0}^{\infty} W(\mathbf{p}, \mathbf{q}) \rho(\mathbf{x}-\mathbf{q}) \sin (2 l \varphi) r \mathrm{~d} r \mathrm{~d} \varphi \mathrm{d} q=\left\langle\psi, \widehat{B}_{2 l}(\mathbf{x}) \psi\right\rangle .
\end{aligned}
$$

Note that the operators $\widehat{A}_{2 l}(\mathbf{x})$ and $\widehat{B}_{2 l}(\mathbf{x})$ depend on the parameter $\mathbf{x}$. Since their symbols are smooth and homogeneous of degree zero in $\mathbf{p}$ they are classical pseudodifferential operators of order zero, see e.g. [44] for the definition of pseudodifferential operators. So we finally obtain the following general expansion of the autocorrelation function

$$
\begin{aligned}
C_{\rho}(\mathbf{x}, \delta \mathbf{x})=\langle & \left., \widehat{A}_{0}(\mathbf{x}) \psi\right\rangle J_{0}(|\delta \mathbf{x}|) \\
& +2 \sum_{l=1}^{\infty}(-1)^{l}\left[\left\langle\psi, \widehat{A}_{2 l}(\mathbf{x}) \psi\right\rangle \cos (2 l \theta)+\left\langle\psi, \widehat{B}_{2 l}(\mathbf{x}) \psi\right\rangle \sin (2 l \theta)\right] J_{2 l}(|\delta \mathbf{x}|) \\
& +O\left(E^{-1 / 2}\right),
\end{aligned}
$$

in terms of the expectation values of a sequence of bounded operators given as Weyl quantizations of the symbols (25). Recall that the only approximation we have made was to insert for $|\mathbf{p}|$ in the exponent in eq. (19) the value at the energy shell $\sqrt{E}$.

Since the Bessel functions have the property that $J_{2 l}(|\delta \mathbf{x}|) \approx 0$ for $|\delta \mathbf{x}| \ll 2 l$, this representation is an efficient expansion for small $|\delta \mathbf{x}|$; the larger $|\delta \mathbf{x}|$ becomes, the more terms of the sum have to be taken into account. Therefore it is desirable to have an estimate on the number of terms which have to be taken into account for large $|\delta \mathbf{x}|$. The first, and largest, maximum of $J_{2 l}(r)$ lies around $r \sim 2 l$, and close to it one has the expansion 43]

$$
J_{2 l}\left(2 l-z l^{1 / 3}\right)=\frac{1}{l^{1 / 3}} \operatorname{Ai}(z)+O(1 / l) .
$$

So the first peak becomes broader with a rate $\sim l^{1 / 3}$ and therefore we have to take for large $r$ approximately

$$
m \sim \frac{r}{2}+\frac{z}{2}\left(\frac{r}{2}\right)^{1 / 3}
$$


terms in the sum over $l$ into account; here $z$ determines the error term. We refer to appendix Q for a more detailed discussion.

We would like to mention two works in which related results have been obtained. In 23 a similar expansion was derived for the case that the eigenfunction is concentrated on an ergodic component of the phase space of a classically mixed system, however without extracting the Bessel function from the expectation values. For the case of a free particle on a surface of constant negative curvature an expansion of the path correlation function in terms of Legendre function was derived in [3]. In the special case of averaging over the whole billiard (i.e. $\rho=1$ ) the path correlation function should be for ergodic systems the same as the autocorrelation function.

The correlation length expansion (28) has various possible applications; some of them will be discussed and illustrated in the next section. In particular, the expansion leads to a prediction for the asymptotic limit of the autocorrelation function in different situations. More precisely, consider a subsequence of eigenfunctions $\left\{\psi_{n_{j}}\right\}_{j \in \mathbb{N}}$ for which the corresponding sequence of Wigner functions converges weakly to a measure $\nu$ on phase space. Such a measure $\nu$ is called a quantum limit, and it is an invariant measure of the classical flow.

If a sequence of eigenfunctions $\left\{\psi_{n_{j}}\right\}_{j \in \mathbb{N}}$ converges to a quantum limit, the correlation length expansion for the autocorrelation function (28) shows that the corresponding sequence of autocorrelation functions converges as well and their limit is obtained by substituting in (28) the expectation values of $\widehat{A}_{2 l}(\mathbf{x})$ and $\widehat{B}_{2 l}(\mathbf{x})$ by their corresponding classical limit. Explicitly, this gives

$$
C_{\rho}^{\operatorname{limit}}(\mathbf{x}, \delta \mathbf{x})=\overline{A_{0}} J_{0}(|\delta \mathbf{x}|)+2 \sum_{l=1}^{\infty}(-1)^{l}\left[\overline{A_{2 l}}(\mathbf{x}) \cos (2 l \theta)+\overline{B_{2 l}}(\mathbf{x}) \sin (2 l \theta)\right] J_{2 l}(|\delta \mathbf{x}|)
$$

where

$$
\bar{A}:=\int_{T^{*} \Omega} A \mathrm{~d} \nu
$$

As we will discuss in section 4.4, for ergodic systems the terms $\overline{A_{2 l}}$ and $\overline{B_{2 l}}$ vanish for $E \rightarrow \infty$, and with $\overline{A_{0}}=1$ we recover $(\overline{12})$.

\section{Applications of the correlation length expansion}

\subsection{Direct comparison}

In the numerical examples we have studied the autocorrelation function in the case $\rho=1$, which allows for an exact computation of the autocorrelation function using the representation (10), which is much more efficient than a direct computation of the autocorrelation function by its definition, eq. (8). In this case the general expansion (28) gives the representation

$$
C(r, \theta)=J_{0}(r)+2 \pi \sum_{l=1}^{\infty}(-1)^{l}\left[a_{2 l} \cos (2 l \theta)+b_{2 l} \sin (2 l \theta)\right] J_{2 l}(r)+O\left(E^{-1 / 2}\right),
$$

where the coefficients $a_{2 l}$ and $b_{2 l}$ are the Fourier coefficients

$$
a_{2 l}=\frac{1}{\pi} \int_{0}^{2 \pi} I(\varphi) \cos (2 l \varphi) \mathrm{d} \varphi \quad b_{2 l}=\frac{1}{\pi} \int_{0}^{2 \pi} I(\varphi) \sin (2 l \varphi) \mathrm{d} \varphi
$$



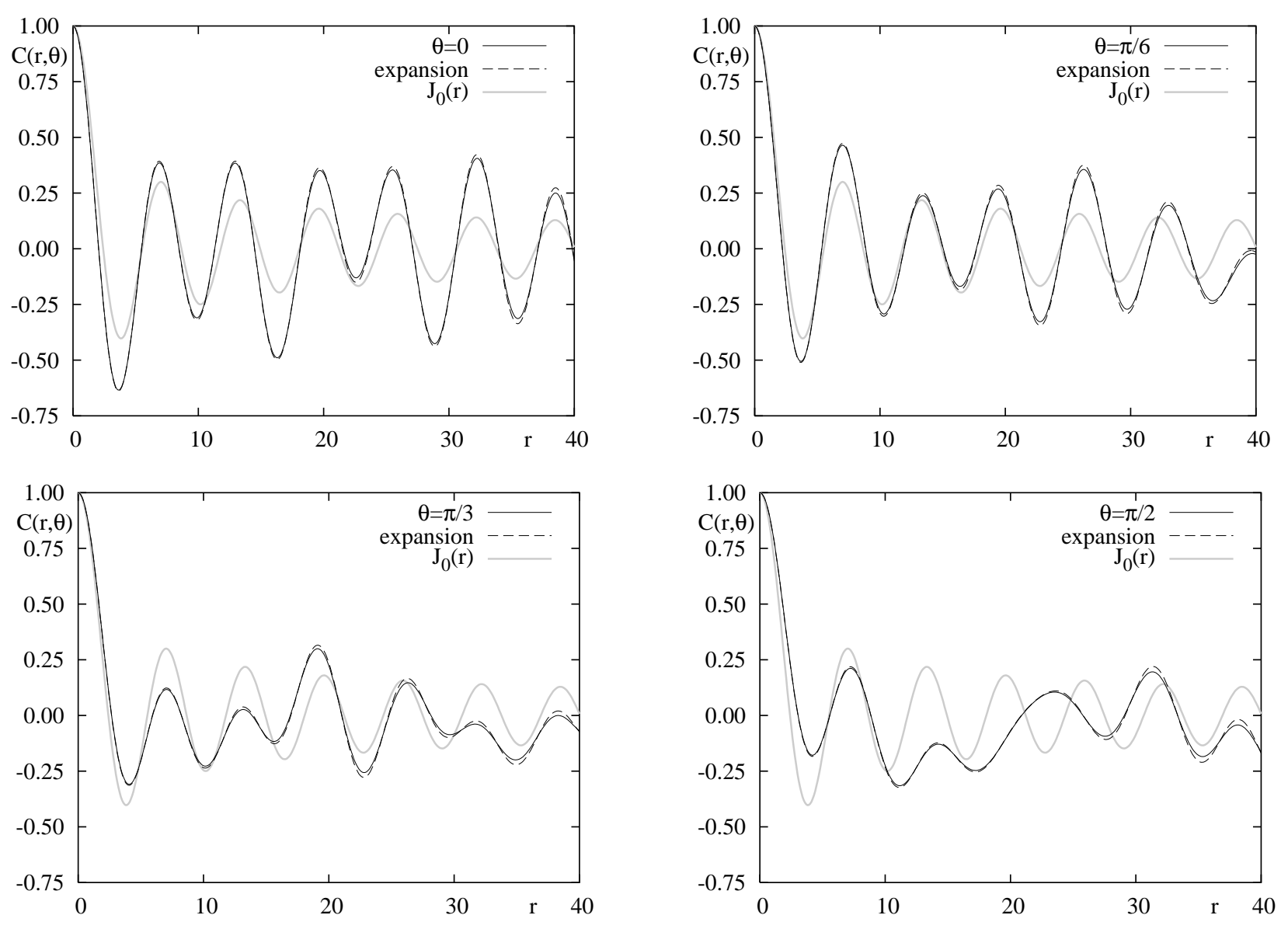

Figure 5: Comparison of the autocorrelation function $C(r, \theta)$ for $\psi_{1907}$ in the stadium billiard (full curve) with the expansion (33). In particular for small $r$ the agreement is excellent, whereas for larger $r$ small differences become visible.

of the radially integrated momentum density [45, 46],

$$
I(\varphi):=\int_{0}^{\infty}|\widehat{\psi}(r \mathbf{e}(\varphi))|^{2} r \mathrm{~d} r,
$$

where $\mathbf{e}(\varphi)=(\cos \varphi, \sin \varphi)$. Also for $I(\varphi)$ a representation in terms of a double integral of the normal derivative function is available [46]. Taking the symmetries into account, one can show that for the odd eigenfunctions in the limaçon billiards and the odd-odd eigenfunctions in the stadium billiard all $b_{2 l}$ vanish, so only the cosine terms remain in (28) and (33).

First we will test the influence of the error term $O\left(E^{-1 / 2}\right)$ in eq. (33) for computations at finite energies. To that end we use the exact quantum $I(\varphi)$ in eq. (34). In fig. 5 the autocorrelation function $C(r, \theta)$ for four different angles $\theta$ is compared to (33). In particular for $r$ not too big the agreement is excellent. Only for larger $r$ do small deviations become visible, which go to zero for higher energies and $r$ fixed. One should remark that for any $r>0$ the effective 

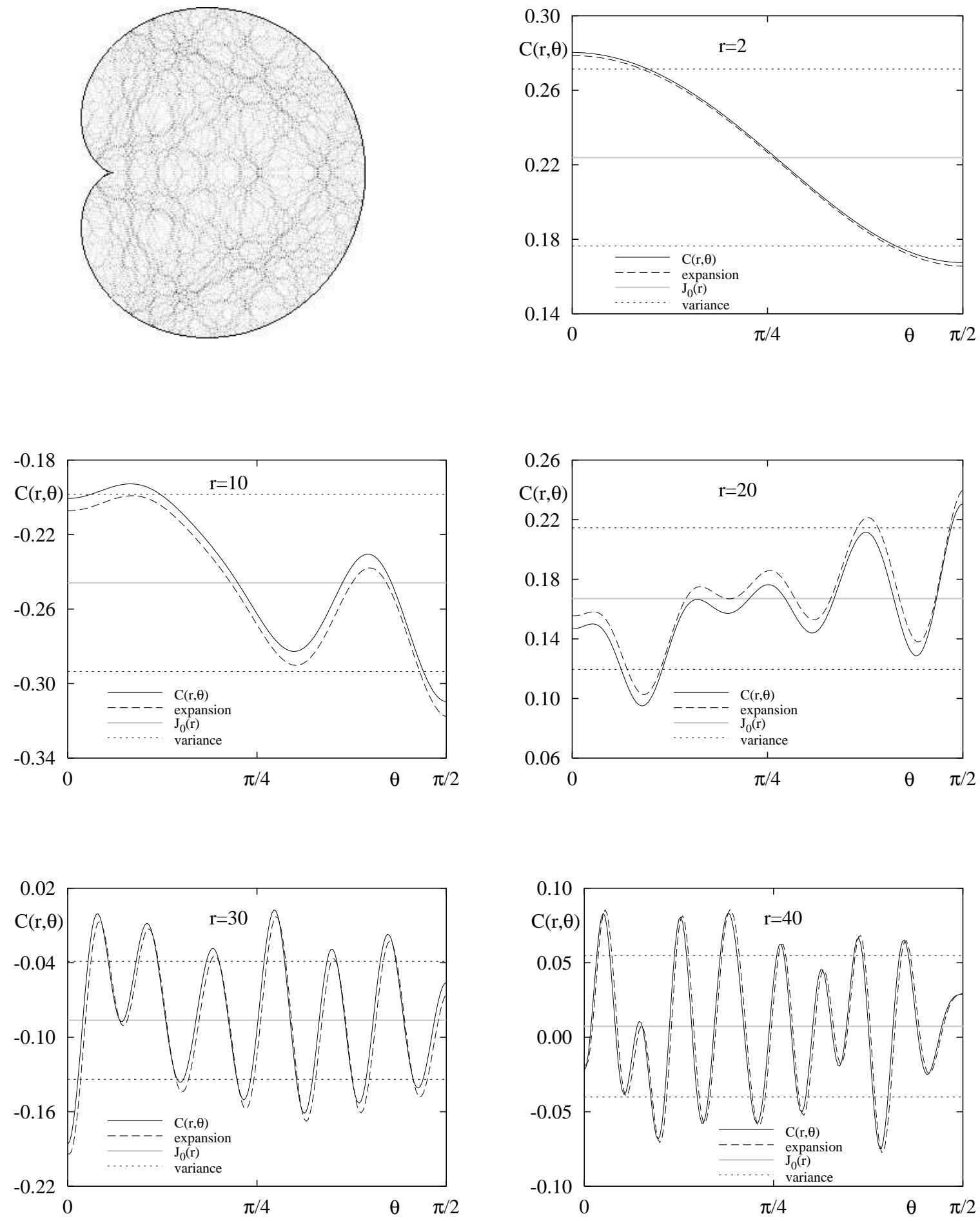

Figure 6: Angular dependence of the autocorrelation function $C(r, \theta)$ for different $r$. Shown are the results for $\psi_{6000}$ in the cardioid billiard with odd symmetry. The full line is the result for $C(r, \theta)$ using (10), the dashed line shows the result of the expansion (33), the full grey line is the value of $J_{0}(r)$ and the dotted horizontal lines show the variance $J_{0}(r) \pm \Delta^{1 / 2}$, see eq. (37). 
integration region in eq. (8) is reduced by the factor

$$
c(r, \theta):=\frac{\operatorname{vol}(\Omega \cap \Omega(r / \sqrt{E}, \theta))}{\operatorname{vol}(\Omega)}
$$

where $\Omega(r / \sqrt{E}, \theta)$ is the set $\Omega$ shifted by the vector $r / \sqrt{E}(\cos \theta, \sin \theta)$. Incorporating this factor leads to an improvement in the agreement of the expansion with the exact autocorrelation function at larger $r$.

Instead of looking at the dependence of the autocorrelation function $C(r, \theta)$ for fixed $\theta$ and varying $r$, it is also interesting to keep $r$ fixed and consider the angular dependence. For a "chaotic" eigenfunction in the cardioid billiard some examples are shown in fig. 6. The result of the expansion (33) is in good agreement with the exact result. For larger $r$ the autocorrelation function $C(r, \theta)$ oscillates more strongly around $J_{0}(r)$. For even larger $r$ we observe clear deviations of the expansion from the exact result (not shown). For comparison the variance of the autocorrelation function around the prediction $J_{0}(r)$ for a random wave model [17] in leading order

$$
\Delta^{1 / 2}=\left(\frac{16}{3 \pi^{3 / 2} A}\right)^{1 / 2} \frac{1}{E^{1 / 4}}
$$

is shown and good agreement is found.

\subsection{Localized eigenfunctions}

For a state strongly localized on an periodic orbit of length $l_{\gamma}$ we have (either in the semiclassical limit, or as a crude model at finite energies)

$$
I(\varphi) \sim \frac{1}{l_{\gamma}} \sum l_{\gamma_{i}} \delta\left(\varphi-\varphi_{i}\right)
$$

where $l_{\gamma_{i}}$ are the lengths of the segments of the orbit with direction $\varphi_{i}$. Thus we get

$$
a_{2 l}=\frac{1}{\pi l_{\gamma}} \sum l_{\gamma_{i}} \cos \left(2 l \varphi_{i}\right) \quad b_{2 l}=\frac{1}{\pi l_{\gamma}} \sum l_{\gamma_{i}} \sin \left(2 l \varphi_{i}\right)
$$

which therefore using (33) gives a prediction for $C(\delta \mathbf{x})$ for such states, namely

$$
C(\delta \mathbf{x}) \sim \frac{1}{l_{\gamma}} \sum_{i} l_{\gamma_{i}} \cos \left(|\delta \mathbf{x}| \cos \left(\theta-\varphi_{i}\right)\right) .
$$

Notice that in the presence of symmetries all symmetry related directions have to be taken into account in eq. (38). For this simple model one can determine the autocorrelation function more directly by using (3)

$$
\begin{aligned}
C(\delta \mathbf{x})=\iint W(\mathbf{p}, \mathbf{q}) \mathrm{e}^{\mathrm{i} \mathbf{p} \delta \mathbf{x}} \mathrm{d} p \mathrm{~d} q & =\int|\widehat{\psi}(\mathbf{p})|^{2} \mathrm{e}^{\mathrm{i} \mathbf{p} \delta \mathbf{x}} \mathrm{d} p \\
& =\int_{0}^{2 \pi} I(\varphi) \cos (|\delta \mathbf{x}| \cos (\theta-\varphi)) \mathrm{d} \varphi+O\left(E^{-1 / 2}\right)
\end{aligned}
$$


inserting (38) directly gives (40).

In fig. 7 we compare the limiting behavior (40) with the autocorrelation function of a high lying eigenstate in the limaçon billiard. The state localizes on the (stable) orbit of triangular shape. Up to $r \approx 10$ the agreement is very good; for larger $r$ the autocorrelation function of the eigenstate shows deviations from the asymptotic behavior. Notice that the state has a much higher energy than the other examples. At lower energies the agreement is not as good, because the region in phase space on which the state localizes is broader. This in turn implies that its corresponding radially integrated momentum distribution $I(\varphi)$ also has broad peaks, which is not accounted for properly by the ansatz (38). However, when considering states of this type with increasing energies, a clear trend to the asymptotic result (40) is observed.

This simple model has also been tested for a scarred state in the cardioid. However, the agreement is limited to a qualitative description for up to $r \approx 2$. This is understandable in view of the observation (see [46, fig. 8a)]) that for a scarred state the radially integrated momentum distribution $I(\varphi)$ shows quite large fluctuations, and also in the considered case the direction $\varphi=\pi / 2$ is not clearly pronounced. As these fluctuations essentially correspond to
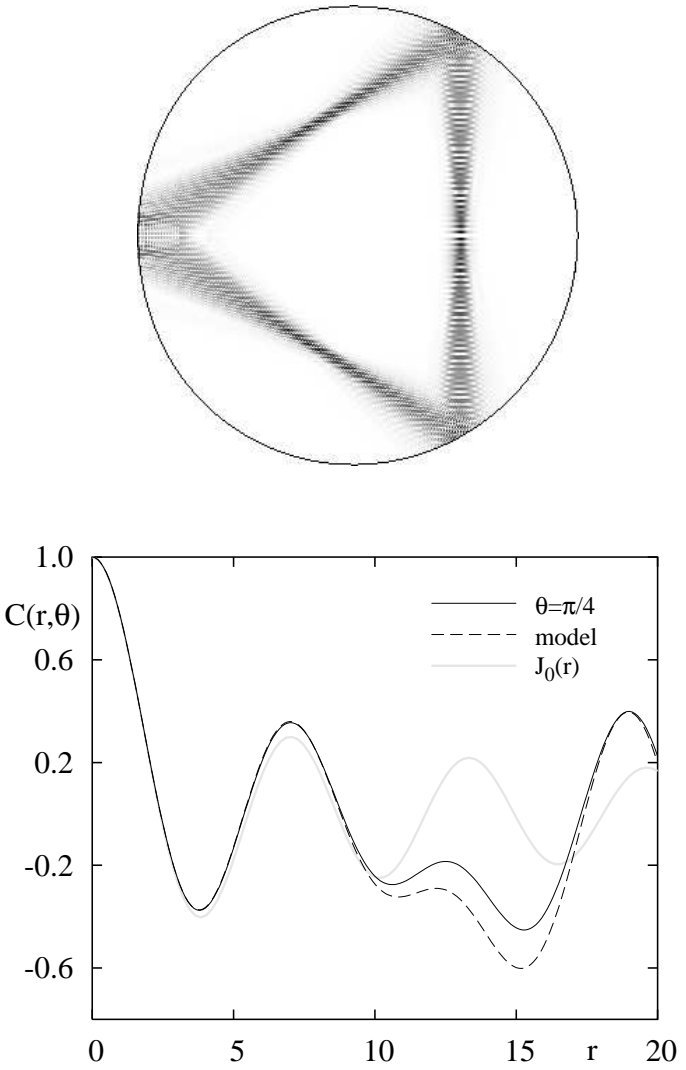
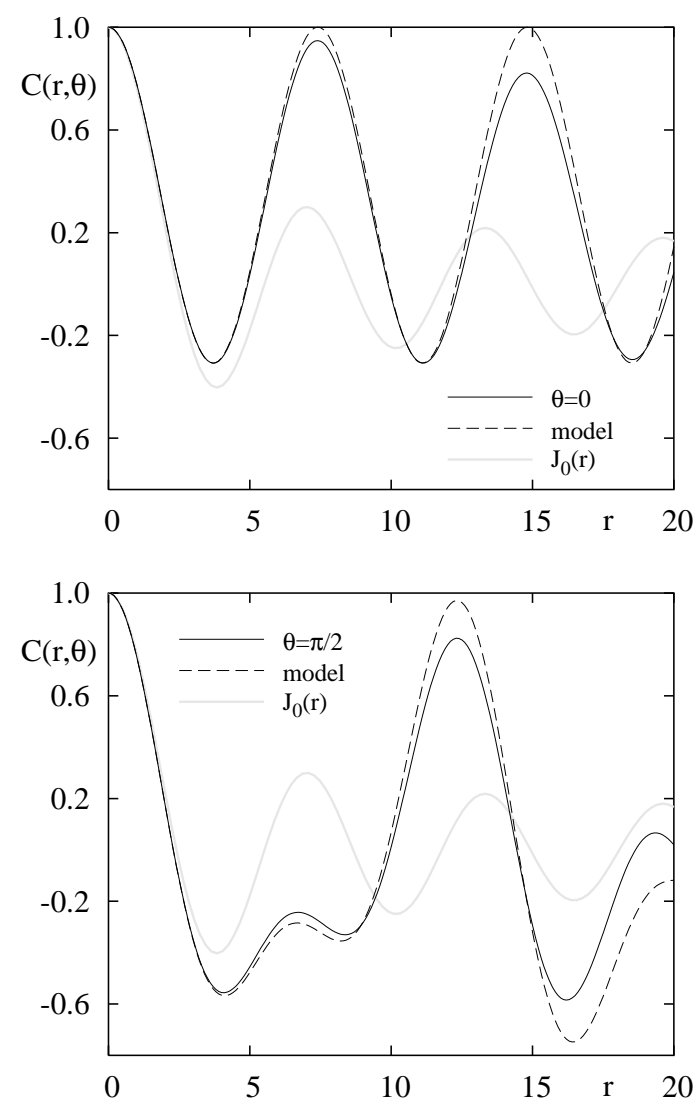

Figure 7: High lying eigenfunction $\left(E=367984.82 \ldots\right.$, approx. $47788^{\text {th }}$ eigenfunction of odd symmetry) in the limaçon billiard $(\varepsilon=0.3)$, which localizes on the stable orbit of triangular shape. The autocorrelation function for three different directions is compared with the $\delta$-model, eq. (40), shown as dashed line using the directions of the stable orbit. 
the random "background" fluctuations of the state, a simple ansatz to model this behavior is

$$
C(r, \theta)=(1-\alpha) J_{0}(r)+\alpha \frac{1}{l_{\gamma}} \sum_{i} l_{\gamma_{i}} \cos \left(|\delta \mathbf{x}| \cos \left(\theta-\varphi_{i}\right)\right) .
$$

It turns out that one can vary $\alpha$ such that quite good agreement of this model with the exact autocorrelation function is obtained, see fig. 8 where $\alpha=0.22$ (for all directions). Depending on the direction $\theta$ the "optimal" value for $\alpha$ does vary, which already indicates the limitations of this simple model. To get a better agreement a more precise description of $I(\varphi)$ for scarred states is necessary. In particular, this should also lead to an understanding of the energy dependence of $\alpha$ which is expected to go to zero in the semiclassical limit. Notice, that the structure of the autocorrelation function is quite similar to the one for $\psi_{1817}$ shown in fig. 3 . Another case, for which we obtain much better agreement, is for an eigenfunction localized on an invariant torus. In such a case the expectation values, eqs. (26), (27), tend to the mean of the classical observable over the torus, see eqs. (31), (32). Fig. 9a) shows for the limaçon billiard the eigenfunction and the corresponding Husimi Poincaré representation [47,48]; see [49] for a more detailed discussion and the formula which has beens used. Also shown in the Husimi plot are the points of some orbits. Using an initial condition on the torus we can determine the classical angular distribution $I^{\text {classical }}(\varphi)$. As this has a singularity due to the caustic of the torus we show in fig. 9v) a binned distribution together with the corresponding quantum radially
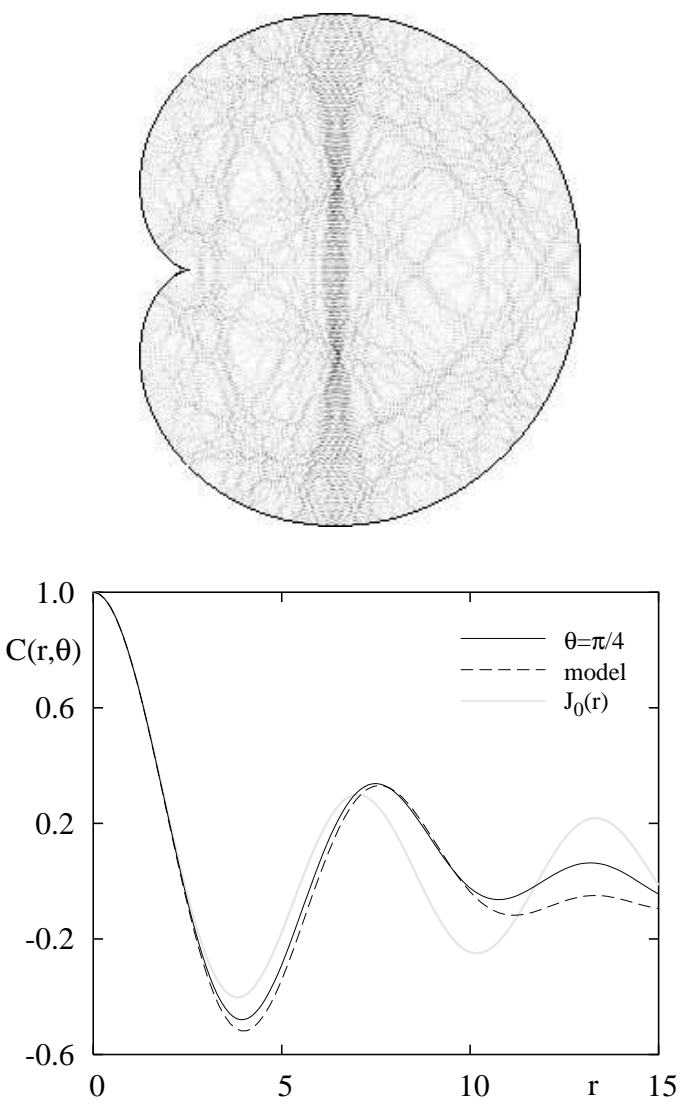
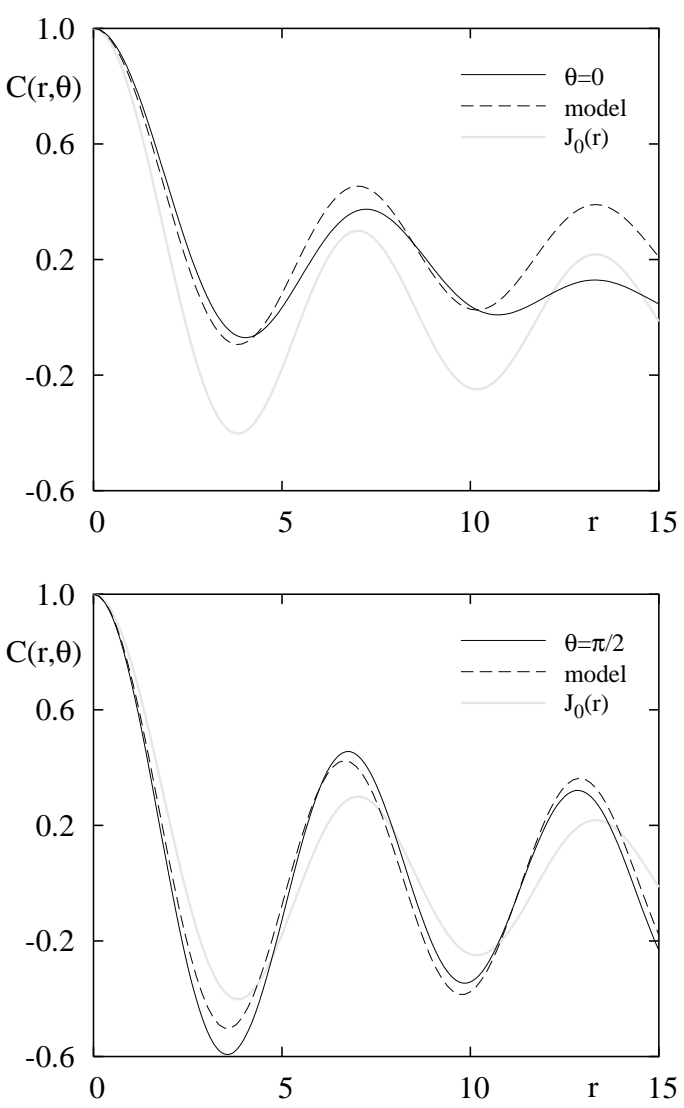

Figure 8: For a scarred state $\left(\psi_{7147}\right.$ of odd symmetry) in the cardioid billiard the autocorrelation function is compared with the simple model (42) for $\alpha=0.22$. 
a)

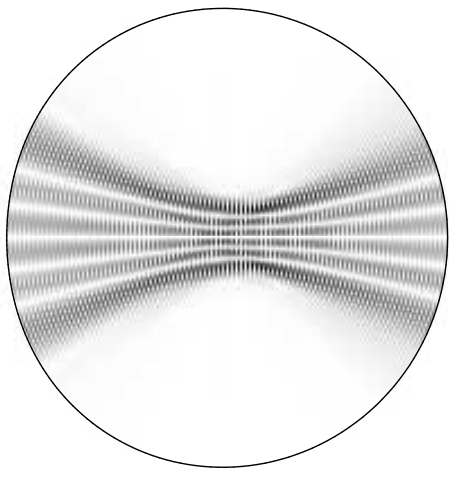

b)

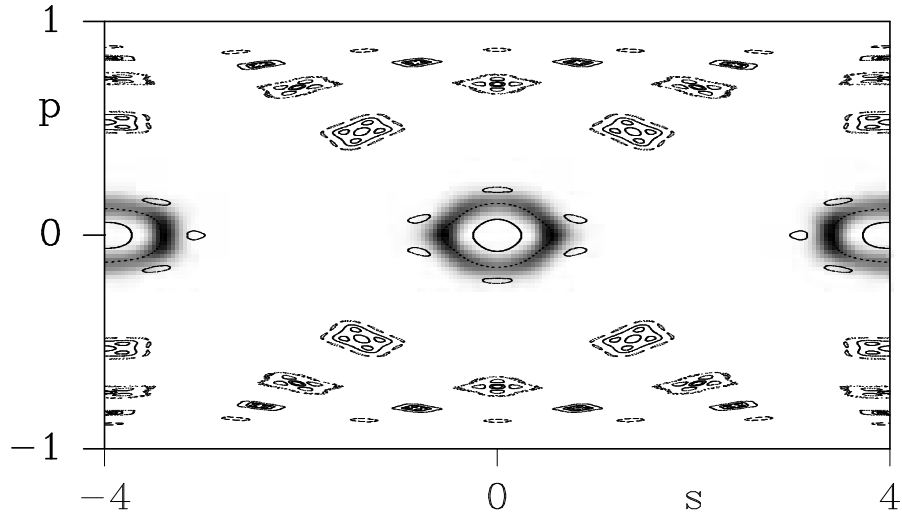

c)

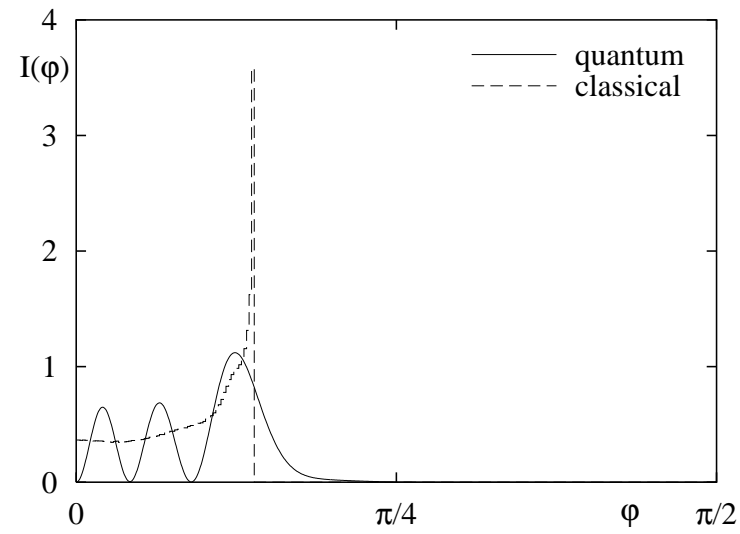

e)

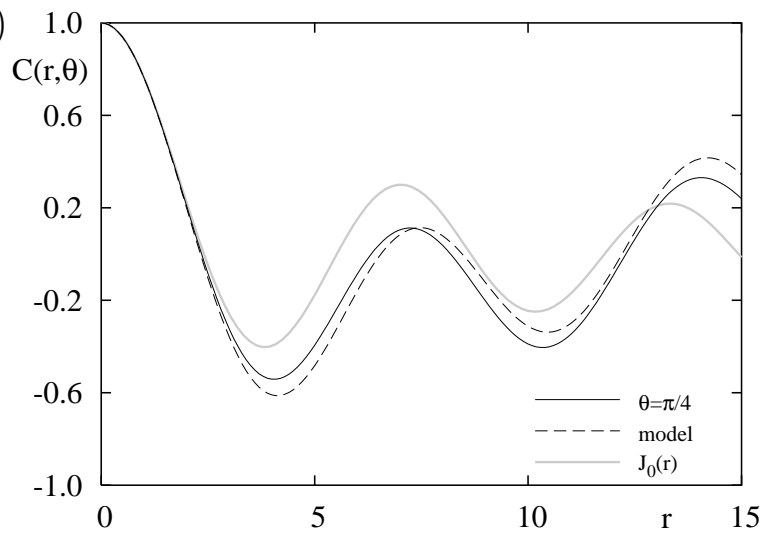

d)

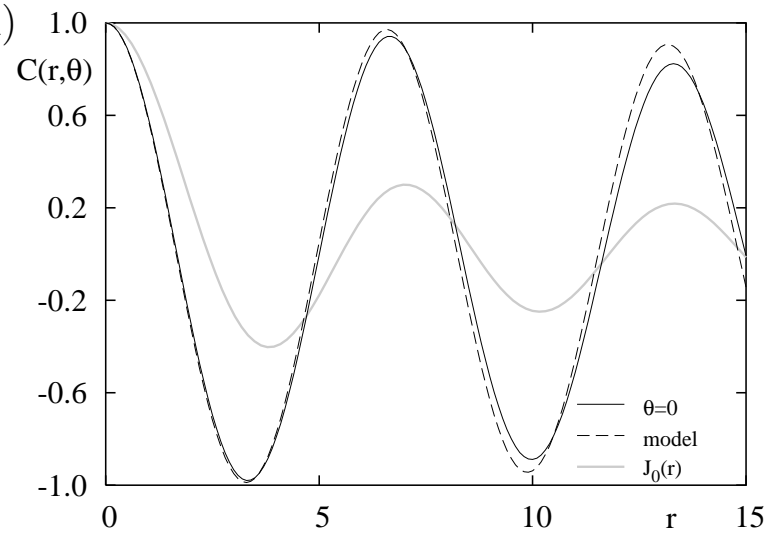

f)

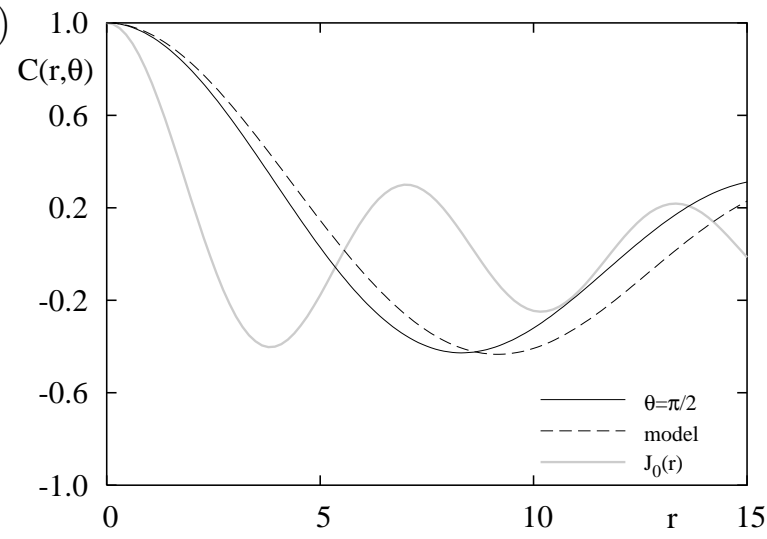

Figure 9: Grey scale plot of $\psi_{3056}$ for the limaçon billiard with $\varepsilon=0.3$ together with the corresponding Husimi plot, for which in addition some orbits are shown. In c) the radially integrated momentum distribution $I_{3056}(\varphi)$ and the corresponding classical distribution $I^{\text {classical }}(\varphi)$ for the torus are shown. In d)-f) the exact autocorrelation function is compared with the expansion of the autocorrelation function, eq. (33), using $I^{\text {classical }}(\varphi)$ for different angles $\theta$. 
integrated momentum distribution $I_{3056}(\varphi)$. There is qualitative agreement between these two curves in the sense that smoothing $I^{\text {classical }}(\varphi)$ describes the mean behavior of the quantum $I_{3056}(\varphi)$. Of course, the classical distribution cannot describe the (quantum) oscillations visible for $I_{3056}(\varphi)$. It turns out, see fig. 9d)-f), that already this simple model leads to surprisingly good agreement between the exact autocorrelation function and the expansion (33) computed using $I^{\text {classical }}(\varphi)$.

\subsection{Autocorrelation function of irregular states in mixed systems}

In classical systems with mixed phase space regions with regular and regions with stochastic behavior coexist. It is conjectured [50] that correspondingly the quantum mechanical eigenfunctions split into regular and irregular ones, respectively, living semiclassically on the correponding parts of phase space. This has been confirmed numerically for several systems, see e.g. [51 55]. Consider now a sequence of eigenfunction $\psi_{n_{j}}$ which localize on some open ergodic domain $D$ in a system with mixed phase space, then almost all the expectation values $\left\langle\psi_{n_{j}} \widehat{A} \psi_{n_{j}}\right\rangle$ tend to

the mean $\bar{A}^{D}$ of the corresponding classical observable $A$ over this domain $D$ [24]. Therefore using (33) we get in the limit $E \rightarrow \infty$ for the autocorrelation function of such a sequence

$$
C_{\rho}^{\text {limit }}(\mathbf{x}, \delta \mathbf{x})={\overline{A_{0}}}^{D} J_{0}(|\delta \mathbf{x}|)+2 \sum_{l=1}^{\infty}(-1)^{l}\left[{\overline{A_{2 l}}}^{D}(\mathbf{x}) \cos (2 l \theta)+{\overline{B_{2 l}}}^{D}(\mathbf{x}) \sin (2 l \theta)\right] J_{2 l}(|\delta \mathbf{x}|)
$$

Instead of computing ${\overline{A_{2 l}}}^{D}$ and ${\overline{B_{2 l}}}^{D}$ directly, we can also use a typical trajectory of the ergodic component to determine the corresponding classical $I^{\text {classical }}(\varphi)$ via

$$
I^{\text {classical }}(\varphi)=\lim _{l \rightarrow \infty} \frac{1}{l} \sum l_{i} \delta\left(\varphi-\varphi_{i}\right)
$$

where $l$ is the total length of the trajectory and $\varphi_{i}$ is the direction of the $i$-th segment having length $l_{i}$. Then we use (33) to get a prediction for the autocorrelation function.

However, we observe that even quite high lying states do not yet localize on the whole chaotic component. Instead they are confined to smaller subregions due to partial barriers in phase space. Fig. 10a) shows an example of a high lying state in the limaçon billiard $(\varepsilon=0.3)$ In fig. 10b) the corresponding Husimi function is plotted, which clearly shows the localization on a chaotic subdomain (the whole irregular region is much larger). If $D$ is a open region in phase space, then the corresponding classical distribution of the momentum directions is given by

$$
I^{\text {classical }}(\varphi)=\frac{1}{\operatorname{vol}(D)} \int \chi_{D}(\mathbf{p}(\varphi), \mathbf{q}) \mathrm{d} q
$$

where $\mathbf{p}(\varphi)=(\cos \varphi, \sin \varphi)$. One can show that in terms of the projection $\mathcal{D}$ of $D$ on the Poincaré section this equation can be reduced to

$$
\begin{aligned}
I^{\text {classical }}(\varphi) & =\frac{\int_{\mathcal{D}} l(s, p) \delta(\varphi-\phi(s, p)) \mathrm{d} s \mathrm{~d} p}{\int_{\mathcal{D}} l(s, p) \mathrm{d} s \mathrm{~d} p} \\
& =\frac{\int_{\partial \Omega^{\prime}(\varphi)} l(s, p(s, \varphi)) \sqrt{1-p^{2}(s, \varphi)} \chi_{\mathcal{D}}(s, p(s, \varphi)) \mathrm{d} s}{\int_{\mathcal{D}} l(s, p) \mathrm{d} s \mathrm{~d} p},
\end{aligned}
$$


a)

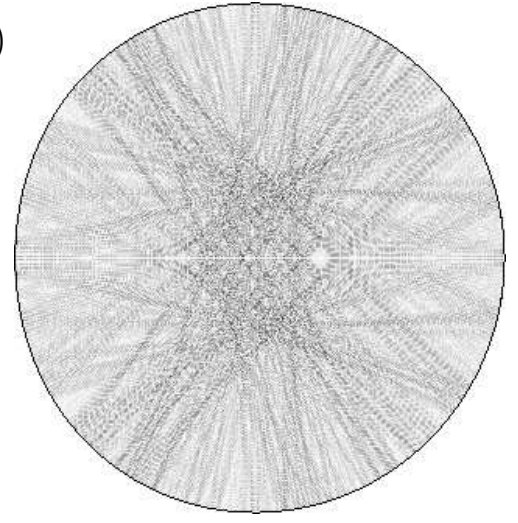

b)

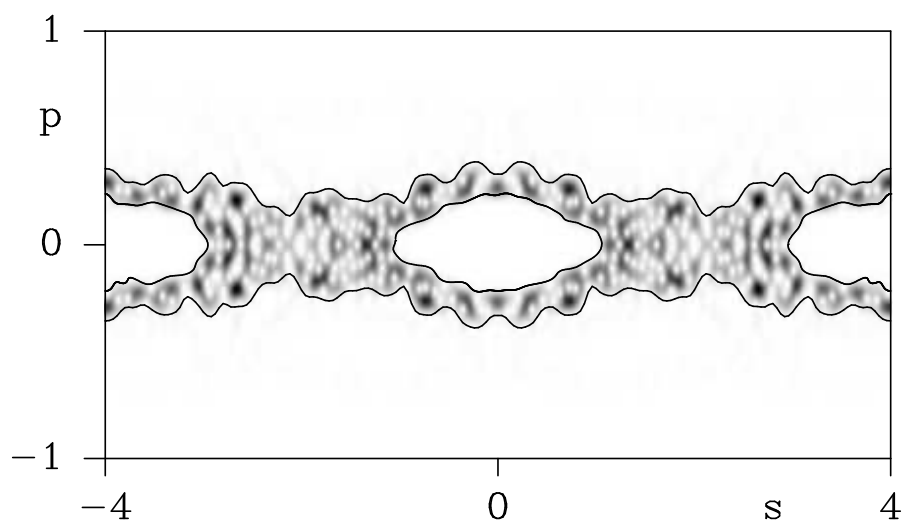

d)

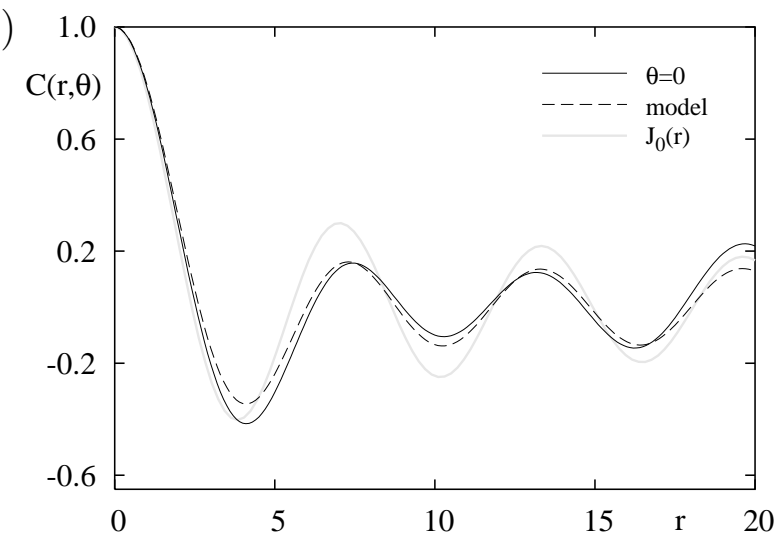

e)

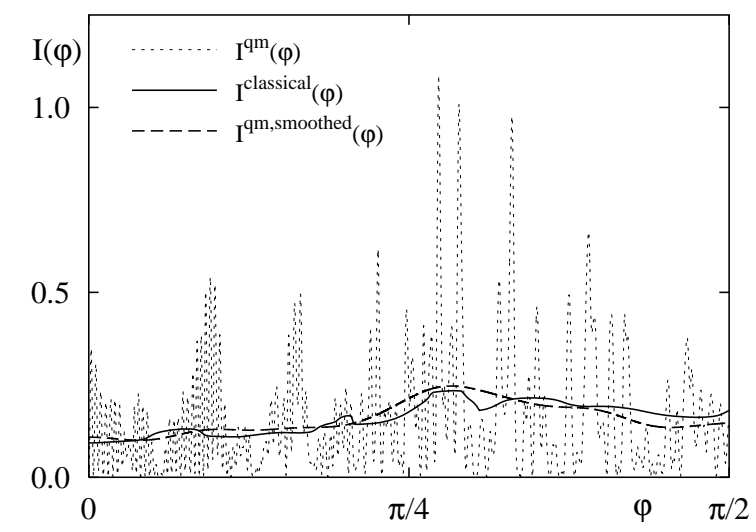

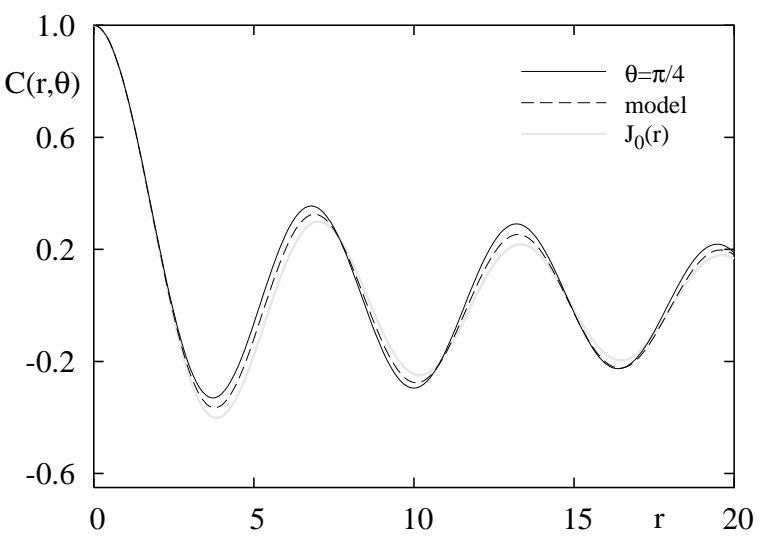

f)

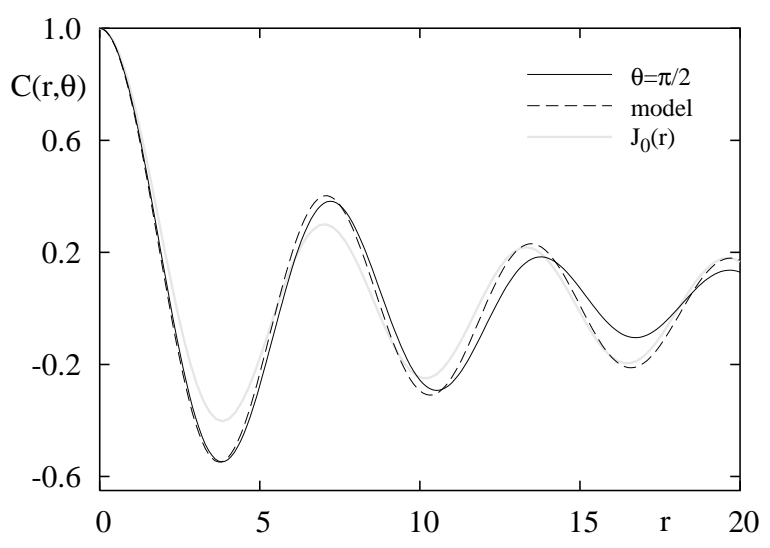

Figure 10: Autocorrelation function for a high lying irregular state $(E=$ $1002754.70 \ldots$, approx. $130516^{\text {th }}$ eigenfunction of odd symmetry) in the limaçon billiard with $\varepsilon=0.3$. In b) the Husimi representation on the boundary is shown together with an approximate boundary (full line) of the region $\mathcal{D}$ on which the state localizes. The resulting classical momentum distribution $I^{\text {classical }}(\varphi)$ is shown in c) as full line and compared with the radially integrated momentum distribution $I^{\mathrm{qm}}(\varphi)$ of the state in a) and a smoothing of this, $I^{\mathrm{qm}, \text { smoothed }}(\varphi)$, shown as dashed line. In $\mathrm{d})-\mathrm{f}$ ) the autocorrelation function $C(r, \theta)$ of the eigenfunction is compared for three different directions with result of the expansion (43) using $I^{\text {classical }}(\varphi)$. 
where $l(s, p)$ is the length of the orbit segment starting in the point $(s, p) \in \mathcal{P}$ with direction $\phi(s, p)$ and in the second equation $p(s, \varphi)=\mathbf{p}(\varphi) \mathbf{t}(s)$, with $\mathbf{t}(s)$ denoting the unit tangent vector to $\partial D$ in the point $s$. Furthermore $\partial \Omega^{\prime}(\varphi):=\{s \in \partial \Omega \mid \mathbf{p}(\varphi) \boldsymbol{n}(s) \leq 0\}$, where $\boldsymbol{n}(s)$ denotes the outer normal vector to $\partial D$ in the point $s$, is the subset of $\partial \Omega$ where the vector $\mathbf{p}(\varphi)$ points inwards. For the numerical computation we have used (47) because we just have to deal with a one-dimensional integral to compute the $\varphi$ dependence, and also compared to (46) no binning of $I^{\text {classical }}(\varphi)$ is necessary.

After these general remarks on the computation of $I^{\text {classical }}(\varphi)$ let us describe how we compute the relevant quantities to determine the autocorrelation function for the state shown in fig. 10a). To describe the projection $\mathcal{D}$ of the domain $D$ in phase space, we use an approximation of the boundary of $\mathcal{D}$ by a splines, which are shown in the fig. 10b) as full curves. Then we use eq. (47) to determine the corresponding $I^{\text {classical }}(\varphi)$, shown in c) as full curve. Of course the radially integrated momentum distribution $I^{\mathrm{qm}}(\varphi)$ of the eigenstate shows strong fluctuations, but the smoothing $I^{\mathrm{qm} \text {,smoothed }}(\varphi)$ is well described by $I^{\text {classical }}(\varphi)$, although the agreement is not perfect. Using $I^{\text {classical }}(\varphi)$ we employ the expansion (43) to get a prediction for the autocorrelation function for states localizing on $\mathcal{D}$, which is compared in figs. $10 \mathrm{~d}-\mathrm{f}$ ) with the exact autocorrelation function. Up to $r \approx 10$ we get quite good agreement, whereas for larger $r$ deviations become more visible. This shows, that the eigenfunction has more structure than accounted for by $I^{\text {classical }}(\varphi)$, i.e. it is not yet far enough in the semiclassical limit.

For higher energies the states tend to localize on the full ergodic region, and then $I^{\text {classical }}(\varphi)$ can simply be computed using (44) by averaging of a typical trajectory in $D$. One should emphasize that the agreement has to be compared with the agreement of the autocorrelation function for ergodic systems with (12) as the prediction eq. (43) only takes into account the classical limit. This has been studied in [23] (in the case of averaging the local autocorrelation function over a small disk), where in particular for [23, Fig. 13b] very good agreement has been found.

\subsection{Ergodic systems and the rate of quantum ergodicity}

If the classical system is ergodic and $\psi_{n_{j}}$ is a quantum ergodic sequence of eigenfunctions, then for $j \rightarrow \infty$

$$
\begin{aligned}
& \left\langle\psi_{n_{j}}, \widehat{A}_{2 l}(\mathbf{x}) \psi_{n_{j}}\right\rangle \sim \overline{A_{2 l}}=\delta_{l 0} \\
& \left\langle\psi_{n_{j}}, \widehat{B}_{2 l}(\mathbf{x}) \psi_{n_{j}}\right\rangle \sim \overline{B_{2 l}}=0 .
\end{aligned}
$$

Thus using the expansion (28) we again get (12) for $E \rightarrow \infty$. Deviations from this universal behavior are then determined by the rate of quantum ergodicity. In order see this it will be convenient to remove the angular dependence by taking the mean over all angular-directions in $C(\delta \mathbf{x})$. Since by eq. (33)

$$
\frac{1}{2 \pi} \int_{0}^{2 \pi} C_{n}(r, \theta) \mathrm{d} \theta=J_{0}(r)+O\left(E^{-1 / 2}\right),
$$

we consider the second moment,

$$
\sigma_{n}^{2}(r):=\frac{1}{2 \pi} \int_{0}^{2 \pi}\left[C_{n}(r, \theta)-J_{0}(r)\right]^{2} \mathrm{~d} \theta
$$


where $C_{n}(r, \theta)$ denotes the autocorrelation function of $\psi_{n}$. Inserting the expansion (33) of $C_{n}(\delta \mathbf{x})$ leads to

$$
\sigma_{n}^{2}(r)=2 \pi^{2} \sum_{l=1}^{\infty}\left(a_{2 l, n}^{2}+b_{2 l, n}^{2}\right)\left[J_{2 l}(r)\right]^{2}\left(1+O\left(E^{-1 / 2}\right)\right) .
$$

In fig. 11 we compare $\sigma^{2}(r)$ for an eigenfunction in the stadium billiard with the expansion (52). For small $r$ we get excellent agreement and some deviations become visible in the plot for $r>20$. The inset shows a plot of the difference up to $r=20$. It is surprising that even though for large $r$ the amplitudes do not match anymore, still the expansion gives the right oscillatory structure.

If we take the mean of (52) over all eigenfunctions up to energy $E$, we get

$$
\begin{aligned}
\overline{\sigma^{2}}(E, r) & :=\frac{1}{N(E)} \sum_{E_{n} \leq E} \sigma_{n}^{2}(r) \\
& =2 \pi^{2} \sum_{l=1}^{\infty} \frac{1}{N(E)} \sum_{E_{n} \leq E}\left(a_{2 l, n}^{2}+b_{2 l, n}^{2}\right)\left[J_{2 l}(r)\right]^{2}\left(1+O\left(E^{-1 / 2}\right)\right)
\end{aligned}
$$

Remarkably, together with eqs. (28) and (52) this shows that the rate of quantum ergodicity can be studied in terms of the autocorrelation function. Particularly interesting is that the

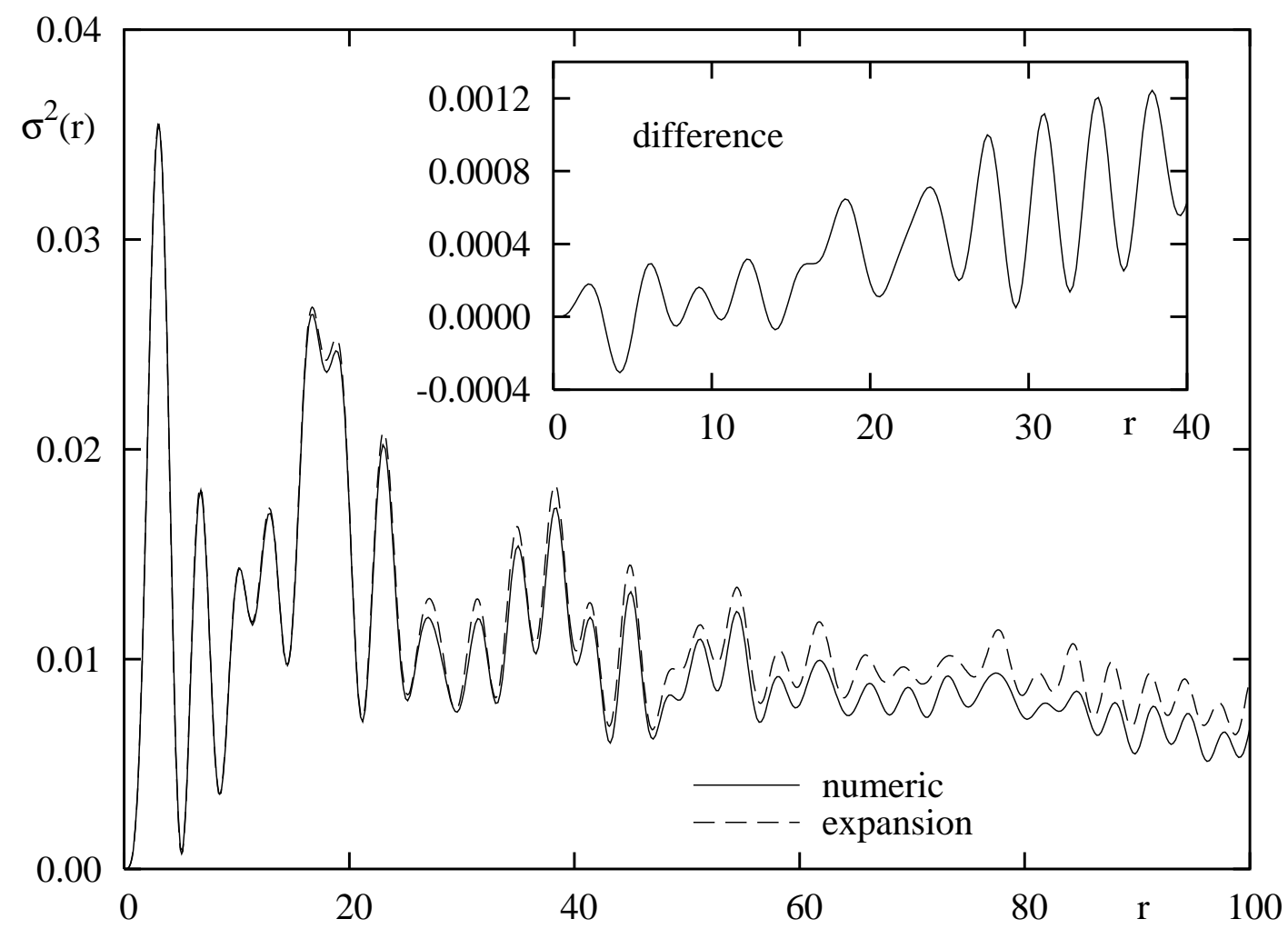

Figure 11: Comparison of the second moment $\sigma_{1907}^{2}(r)$ of the autocorrelation function, eq. (51), with the expansion (52) for the stadium billiard. The inset shows the difference for $r \in[0,20]$. 
observables in the expansion (28) become more and more oscillatory with increasing $l$, so by varying $|\delta \mathbf{x}|$ one can determine the rate of quantum ergodicity on different length scales.

A prediction for the behavior of $\overline{\sigma^{2}}(E, r)$ follows from [56], where it is shown that (under suitable conditions on the system) in the mean

$$
\frac{1}{N(E)} \sum_{E_{n} \leq E}\left[\left\langle\psi_{n}, \widehat{A} \psi_{n}\right\rangle-\bar{A}\right]^{2} \sim \frac{4 \sigma_{\mathrm{cl}}^{2}(A)}{\operatorname{vol} \Omega} \frac{1}{\sqrt{E}}
$$

for any pseudodifferential operator $\widehat{A}$ of order zero with symbol $A$. Here $\bar{A}$ denotes the mean value of $A$, and $\sigma_{\mathrm{cl}}^{2}(A) / \sqrt{T}$ is the variance of the fluctuations of

$$
\frac{1}{T} \int_{0}^{T} A(\mathbf{p}(t), \mathbf{q}(t)) \mathrm{d} t
$$

around $\bar{A}$. So if we insert (55) into (54) we obtain

$$
\overline{\sigma^{2}}(E, r) \sim \frac{8 \pi^{2}}{\operatorname{vol} \Omega} \sum_{l=1}^{\infty}\left[\sigma_{\mathrm{cl}}^{2}\left(A_{2 l}\right)+\sigma_{\mathrm{cl}}^{2}\left(B_{2 l}\right)\right]\left[J_{2 l}(r)\right]^{2} \frac{1}{\sqrt{E}} .
$$

A detailed study of the rate of quantum ergodicity in terms of the autocorrelation function, i.e. via eq. (53), and a comparison with the semiclassical expectation (57) will be given in a separate paper.

\section{Summary}

We have discussed the autocorrelation function for eigenstates of quantum mechanical systems, and its relation to the behavior of the classical system. For billiards we have derived a formula for the autocorrelation function of an eigenfunction in terms of the normal derivative on the boundary (10), which enables an efficient numerical computation.

Our main result is the correlation length expansion of the autocorrelation function (28), which provides an efficient expansion for small correlation length, where only a small number of terms enter. Moreover, it provides a tool for understanding the behavior of the autocorrelation function for different types of eigenfunctions in terms of their semiclassical limit.

The coefficients in the correlation length expansion (28) can be computed in terms of the radially integrated momentum density. Even though it is based on an approximation, our numerical study shows very good agreement with the corresponding exact results; only for large correlation lengths deviations become visible. As the expansion coefficients have to be determined just once for a given eigenfunction, this is also a numerically efficient method to compute the autocorrelation function. Similar expansions can be derived in higher dimension and for more general systems (e.g. systems with potential and magnetic field), but then the Bessel functions have to be modified in order to reflect the structure of the energy shell of the classical system.

We applied the expansion of the autocorrelation function to different types of eigenfunctions, and showed that it provides a good tool for the understanding of their autocorrelation functions. In systems with mixed phase space regular states concentrated on tori and irregular 
states have been successfully treated. For chaotic system the fluctuations of the autocorrelation functions around the leading term are shown to be connected with the rate of quantum ergodicity. Moreover by varying the correlation length the autocorrelation function is shown to be an interesting new tool to measure the rate of quantum ergodicity on different length scales.

\section{Acknowledgements}

We would like to thank Professor Jonathan Keating for useful comments on the manuscript. A.B. acknowledges support by the Deutsche Forschungsgemeinschaft under contract No. DFGBa 1973/1-1. R.S. acknowledges support by the Deutsche Forschungsgemeinschaft under contract No. DFG-Ste 241/7-3.

\section{Appendix}

\section{A Autocorrelation function in terms of normal deriva- tives on the boundary}

We will give a derivation of the formula (10) which provides an expression of the autocorrelation function $C(\delta \mathbf{x})$ in terms of the normal derivative. Let $\psi(\mathbf{q})$ be a solution of the Helmholtz equation with Dirichlet boundary condition on $\partial \Omega$,

$$
\left(\Delta+k^{2}\right) \psi(\mathbf{q})=0, \quad \psi(\mathbf{q})=0 \quad \text { for } \mathbf{q} \in \partial \Omega,
$$

where we have defined $k=\sqrt{E}$, and let

$$
u(s):=\boldsymbol{n}(s) \nabla \psi(\mathbf{q}(s))
$$

be the outer normal derivative of $\psi$ on $\partial \Omega$, where $s$ parameterizes $\partial \Omega$ in arclength. It is well known that

$$
-\frac{1}{4} \int_{\partial \Omega} Y_{0}(k|\mathbf{q}-\mathbf{q}(s)|) u(s) \mathrm{d} s= \begin{cases}\psi(\mathbf{q}) & \text { for } \mathbf{q} \in \stackrel{\circ}{\Omega} \\ 0 & \text { for } \mathbf{q} \notin \Omega\end{cases}
$$

and furthermore

$$
\int_{\partial \Omega} J_{0}(k|\mathbf{q}-\mathbf{q}(s)|) u(s) \mathrm{d} s=0 .
$$

Let $\rho(t)$ be a smooth cut-off function with

$$
\rho(k t)=\left\{\begin{array}{ll}
1 & \text { for } t \leq 2 \operatorname{diam}(\Omega) \\
0 & \text { for } t \geq 3 \operatorname{diam}(\Omega)
\end{array},\right.
$$

where $\operatorname{diam}(\Omega)$ denotes the diameter of $\Omega$. Then we have for $\mathbf{q}$ in some neighborhood of $\Omega$

$$
\psi(\mathbf{q})=-\frac{1}{4} \int_{\partial \Omega} \rho(k|\mathbf{q}-\mathbf{q}(s)|) Y_{0}(k|\mathbf{q}-\mathbf{q}(s)|) u(s) \mathrm{d} s
$$


and obtain

$$
C(\delta \mathbf{x})=\int_{\mathbb{R}^{2}} \psi^{*}(\mathbf{q}) \psi(\mathbf{q}+\delta \mathbf{x}) \mathrm{d} q=\iint_{\partial \Omega \times \partial \Omega} K_{\rho}\left(\delta \mathbf{x}, s, s^{\prime}\right) u^{*}(s) u\left(s^{\prime}\right) \mathrm{d} s \mathrm{~d} s^{\prime},
$$

with

$$
\begin{aligned}
K_{\rho}\left(\delta \mathbf{x}, s, s^{\prime}\right) & =\frac{1}{16} \int_{\mathbb{R}^{2}} \rho(k|\mathbf{q}-\mathbf{q}(s)|) Y_{0}(k|\mathbf{q}-\mathbf{q}(s)|) Y_{0}\left(k\left|\mathbf{q}-\mathbf{q}\left(s^{\prime}\right)+\delta \mathbf{x}\right|\right) \mathrm{d} q \\
& =\frac{1}{16} \int_{\mathbb{R}^{2}} \rho(k|\mathbf{q}|) Y_{0}(k|\mathbf{q}|) Y_{0}\left(k\left|\mathbf{q}+\mathbf{q}(s)-\mathbf{q}\left(s^{\prime}\right)+\delta \mathbf{x}\right|\right) \mathrm{d} q .
\end{aligned}
$$

Due to the factor $\rho(k|\mathbf{q}-\mathbf{q}(s)|)$ this integral is absolutely convergent. We now use Grafs addition theorem 43

$$
Y_{0}(k|\mathbf{q}+\Delta \mathbf{q}|)= \begin{cases}\sum_{l \in \mathbb{Z}} Y_{l}(k|\Delta \mathbf{q}|) J_{l}(k|\mathbf{q}|) \cos (l \varphi) & \text { for }|\mathbf{q}|<|\Delta \mathbf{q}| \\ \sum_{l \in \mathbb{Z}} Y_{l}(k|\mathbf{q}|) J_{l}(k|\Delta \mathbf{q}|) \cos (l \varphi) & \text { for }|\mathbf{q}|>|\Delta \mathbf{q}|\end{cases}
$$

where $\Delta \mathbf{q}=\mathbf{q}(s)-\mathbf{q}\left(s^{\prime}\right)+\delta \mathbf{x}$ and $\varphi$ is the angle between $\Delta \mathbf{q}$ and $\mathbf{q}$. Introducing polar coordinates in the integral in (65) and using (66) gives

$$
\begin{aligned}
K_{\rho}\left(\delta \mathbf{x}, s, s^{\prime}\right)= & \frac{\pi}{8} \int_{0}^{|\Delta \mathbf{q}|} Y_{0}(k r) J_{0}(k r) r \mathrm{~d} r Y_{0}(k|\Delta \mathbf{q}|) \\
& +\frac{\pi}{8} \int_{|\Delta \mathbf{q}|}^{\infty} \rho(k r) Y_{0}(k r) Y_{0}(k r) r \mathrm{~d} r J_{0}(k|\Delta \mathbf{q}|)
\end{aligned}
$$

where we have furthermore used that $\rho(k r)=1$ for $r \leq|\Delta \mathbf{q}|$ by (62). The first integral is

$$
\int_{0}^{|\Delta \mathbf{q}|} Y_{0}(k r) J_{0}(k r) r \mathrm{~d} r=\frac{|\Delta \mathbf{q}|^{2}}{2}\left[Y_{0}(k|\Delta \mathbf{q}|) J_{0}(k|\Delta \mathbf{q}|)+Y_{1}(k|\Delta \mathbf{q}|) J_{1}(k|\Delta \mathbf{q}|)\right],
$$

see e.g. 433, and for the second one partial integration gives

$$
\begin{aligned}
\int_{|\Delta \mathbf{q}|}^{\infty} \rho(k r) Y_{0}(k r) Y_{0}(k r) r \mathrm{~d} r=- & \frac{|\Delta \mathbf{q}|^{2}}{2}\left[Y_{0}(k|\Delta \mathbf{q}|) Y_{0}(k|\Delta \mathbf{q}|)+Y_{1}(k|\Delta \mathbf{q}|) Y_{1}(k|\Delta \mathbf{q}|)\right] \\
& -\frac{k}{2} \int_{|\Delta \mathbf{q}|}^{\infty} \rho^{\prime}(k r)\left[Y_{0}(k r) Y_{0}(k r)+Y_{1}(k r) Y_{1}(k r)\right] r^{2} \mathrm{~d} r .
\end{aligned}
$$

Note that since $\rho^{\prime}$ has compact support the second integral is over a finite interval, and for $s, s^{\prime} \in \partial \Omega, \delta \mathbf{x} \in \Omega$ the lower limit of the integral, $|\Delta \mathbf{q}|$, is outside the support of $\rho^{\prime}$, hence the second term on the right hand side of eq. (69) is constant. So we get

$$
K_{\rho}\left(\delta \mathbf{x}, s, s^{\prime}\right)=K\left(\delta \mathbf{x}, s, s^{\prime}\right)+R_{\rho}\left(\delta \mathbf{x}, s, s^{\prime}\right)
$$


with

$$
K\left(\delta \mathbf{x}, s, s^{\prime}\right)=\frac{\pi|\Delta \mathbf{q}|^{2}}{16}\left[Y_{1}(k|\Delta \mathbf{q}|) J_{1}(k|\Delta \mathbf{q}|) Y_{0}(k|\Delta \mathbf{q}|)-Y_{1}(k|\Delta \mathbf{q}|) Y_{1}(k|\Delta \mathbf{q}|) J_{0}(k|\Delta \mathbf{q}|)\right]
$$

and

$$
R_{\rho}\left(\delta \mathbf{x}, s, s^{\prime}\right)=C J_{0}(k|\Delta \mathbf{q}|)
$$

with $C$ constant and by (61) this term gives no contribution to $C(\delta \mathbf{x})$. Using a Wronsky determinant of Bessel functions [43] we can simplify $K\left(\delta \mathbf{x}, s, s^{\prime}\right)$ further

$$
\begin{aligned}
K\left(\delta \mathbf{x}, s, s^{\prime}\right) & =\frac{\pi|\Delta \mathbf{q}|^{2}}{16} Y_{1}(k|\Delta \mathbf{q}|)\left[J_{1}(k|\Delta \mathbf{q}|) Y_{0}(k|\Delta \mathbf{q}|)-Y_{1}(k|\Delta \mathbf{q}|) J_{0}(k|\Delta \mathbf{q}|)\right] \\
& =\frac{\pi|\Delta \mathbf{q}|^{2}}{16} Y_{1}(k|\Delta \mathbf{q}|) \frac{2}{\pi k|\Delta \mathbf{q}|}=\frac{|\Delta \mathbf{q}|}{8 k} Y_{1}(k|\Delta \mathbf{q}|)
\end{aligned}
$$

which gives the final result.

\section{B Remainder estimate}

In this appendix we sketch the derivation of the remainder estimate in equation (19). We start by representing the integral as an expectation value, see (24),

$$
\int_{0}^{\infty} \int_{0}^{2 \pi} \int_{\Omega} \rho(\mathbf{x}-\mathbf{q}) W(\mathbf{p}, \mathbf{q}) \mathrm{d} q^{\prime} \mathrm{e}^{\mathrm{i} r|\delta \mathbf{x}| \cos (\varphi-\theta) / \sqrt{E}} r \mathrm{~d} \varphi \mathrm{d} r=\langle\psi, A \psi\rangle
$$

where $A$ is the Weyl quantization of the symbol

$$
a(\mathbf{p}, \mathbf{q}):=\rho(\mathbf{x}-\mathbf{q}) \mathrm{e}^{\mathrm{i} \frac{|\mathbf{p}|}{\sqrt{E}}|\delta \mathbf{x}| \cos (\varphi-\theta)} .
$$

The basic idea is to find a decomposition of the operator $A$,

$$
A=A_{0}+(\sqrt{-\Delta}-\sqrt{E}) A_{1}+R
$$

where $A_{0}$ has Weyl symbol

$$
a_{0}(\mathbf{p}, \mathbf{q})=\rho(\mathbf{x}-\mathbf{q}) \mathrm{e}^{\mathrm{i}|\delta \mathbf{x}| \cos (\varphi-\theta)}
$$

and the remainder $R$ satisfies

$$
\|R\| \leq C E^{-1 / 2}
$$

If we assume the decomposition (76) and take the expectation value of both sides, one gets

$$
\langle\psi, A \psi\rangle=\left\langle\psi, A_{0} \psi\right\rangle+\langle\psi, R \psi\rangle
$$


where $(\sqrt{-\Delta}-\sqrt{E}) \psi=0$ has been used. In terms of the symbols eq. (79) is the desired result, see (19),

$$
\begin{aligned}
\int_{0}^{\infty} \int_{0}^{2 \pi} \int_{\Omega} W(\mathbf{p}, \mathbf{q}) \rho(\mathbf{x}-\mathbf{q}) \mathrm{d} q^{\prime} \mathrm{e}^{\mathrm{i} r|\delta \mathbf{x}| \cos (\varphi-\theta) / \sqrt{E}} r \mathrm{~d} \varphi \mathrm{d} r \\
=\int_{0}^{\infty} \int_{0}^{2 \pi} \int_{\Omega} W(\mathbf{p}, \mathbf{q}) \rho(\mathbf{x}-\mathbf{q}) \mathrm{d} q^{\prime} \mathrm{e}^{\mathrm{i}|\delta \mathbf{x}| \cos (\varphi-\theta) /} r \mathrm{~d} \varphi \mathrm{d} r+O\left(E^{-1 / 2}\right) .
\end{aligned}
$$

Let us now show, that the decomposition (76) is basically a quantization of the Taylor expansion of the symbol $a(\mathbf{p}, \mathbf{q})$ around $|\mathbf{p}|=\sqrt{E}$,

$$
a(\mathbf{p}, \mathbf{q})=a_{0}(\mathbf{p}, \mathbf{q})+(|\mathbf{p}|-\sqrt{E}) a_{1}(\mathbf{p}, \mathbf{q})
$$

Quantizing this classical decomposition yields (76) with $R$ given as Weyl quantization of

$$
r(\mathbf{p}, \mathbf{q})=(|\mathbf{p}|-\sqrt{E}) a_{1}(\mathbf{p}, \mathbf{q})-(|\mathbf{p}|-\sqrt{E}) \# a_{1}(\mathbf{p}, \mathbf{q})
$$

since the Weyl symbol of $(\sqrt{-\Delta}-\sqrt{E}) A_{1}$ is $(|\mathbf{p}|-\sqrt{E}) \# a_{1}(\mathbf{p}, \mathbf{q})$ with \# denoting the symbol product, see e.g. 44]. Since $E$ is a constant we have

$$
r(\mathbf{p}, \mathbf{q})=|\mathbf{p}| a_{1}(\mathbf{p}, \mathbf{q})-|\mathbf{p}| \# a_{1}(\mathbf{p}, \mathbf{q})
$$

and this is a function which is bounded and of order $O\left(E^{-1 / 2}\right)$, and all its derivatives are bounded and of order $O\left(E^{-1 / 2}\right)$, too. So by the Calderon Vallaincourt theorem [44 the estimate (78) follows.

\section{Estimating the Bessel sum}

In this appendix we determine how many terms in the sum (28) have to be taken into account such that the remainder is smaller than some given error $\delta$. From (26) and (27) it follows that for fixed $\psi$

$$
\left|\left\langle\psi, \widehat{A}_{2 l}(\mathbf{x}) \psi\right\rangle \cos (2 l \theta)+\left\langle\psi, \widehat{B}_{2 l}(\mathbf{x}) \psi\right\rangle \sin (2 l \theta)\right| \leq C
$$

Thus if we split the sum

$$
\begin{aligned}
& \sum_{l=1}^{\infty}(-1)^{l}\left[\left\langle\psi, \widehat{A}_{2 l}(\mathbf{x}) \psi\right\rangle \cos (2 l \theta)+\left\langle\psi, \widehat{B}_{2 l}(\mathbf{x}) \psi\right\rangle \sin (2 l \theta)\right] J_{2 l}(|\delta \mathbf{x}|) \\
& \quad=\sum_{l=1}^{m-1}(-1)^{l}\left[\left\langle\psi, \widehat{A}_{2 l}(\mathbf{x}) \psi\right\rangle \cos (2 l \theta)+\left\langle\psi, \widehat{B}_{2 l}(\mathbf{x}) \psi\right\rangle \sin (2 l \theta)\right] J_{2 l}(|\delta \mathbf{x}|)+R_{m}(|\delta \mathbf{x}|)
\end{aligned}
$$

we get for the remainder

$$
\left|R_{m}(r)\right| \leq C \sum_{l=m}^{\infty}\left|J_{2 l}(r)\right|
$$


Therefore we have to estimate the sum over Bessel functions

$$
\sum_{l=m}^{\infty}\left|J_{2 l}(r)\right|
$$

and determine its dependence on $m$ and $r$. The asymptotics in the transition region

$$
J_{2 l}\left(2 l-z(2 l)^{1 / 3}\right) \sim \frac{1}{l^{1 / 3}} \operatorname{Ai}\left(2^{1 / 3} z\right)
$$

gives that $J_{2 l}(r)$ is monotonically increasing for $r<2 l$, such that for $r<2 m$

$$
\sum_{l=m}^{\infty}\left|J_{2 l}(r)\right|=\sum_{l=m}^{\infty} \frac{1}{l^{1 / 3}} \mathrm{Ai}\left(\frac{2 l-r}{l^{1 / 3}}\right)+O\left(m^{-1}\right) .
$$

Defining $z$ by

$$
r=2 m-z m^{1 / 3}
$$

we obtain

$$
\begin{aligned}
\sum_{l=m}^{\infty} \frac{1}{l^{1 / 3}} \mathrm{Ai}\left(\frac{2 l-r}{l^{1 / 3}}\right) & =\sum_{l=m}^{\infty} \frac{1}{l^{1 / 3}} \mathrm{Ai}\left(\frac{2(l-m)}{l^{1 / 3}}+z\left(\frac{m}{l}\right)^{1 / 3}\right) \\
& =\sum_{l=0}^{\infty} \frac{1}{(l+m)^{1 / 3}} \mathrm{Ai}\left(\frac{2 l}{(l+m)^{1 / 3}}+z\left(\frac{m}{l+m}\right)^{1 / 3}\right) \\
& =\sum_{l=0}^{\infty} \frac{1}{m^{1 / 3}} \mathrm{Ai}\left(\frac{2 l}{m^{1 / 3}}+z\right)+O\left(m^{-1 / 3}\right)
\end{aligned}
$$

where we have furthermore used that for large $m$ only the terms with $l \ll m$ contribute, because the Airy function is exponentially decreasing for positive arguments. The Euler McLaurin formula then gives

$$
\begin{aligned}
\sum_{l=0}^{\infty} \frac{1}{m^{1 / 3}} \mathrm{Ai}\left(\frac{2 l}{m^{1 / 3}}+z\right) & =\int_{0}^{\infty} \frac{1}{m^{1 / 3}} \mathrm{Ai}\left(\frac{2 l}{m^{1 / 3}}+z\right) \mathrm{d} l+O\left(m^{-1 / 3}\right) \\
& =\frac{1}{2} \int_{z}^{\infty} \operatorname{Ai}(x) \mathrm{d} x+O\left(m^{-1 / 3}\right)
\end{aligned}
$$

And so finally we arrive at

$$
\sum_{l=m}^{\infty}\left|J_{2 l}(r)\right|=\frac{1}{2} \int_{z}^{\infty} \operatorname{Ai}(x) \mathrm{d} x+O\left(m^{-1 / 3}\right) .
$$

The function $\int_{z}^{\infty} \operatorname{Ai}(x) \mathrm{d} x$ is monotonically decreasing, so for a given $\delta>0$ we can define a $z(\delta)$ by

$$
\frac{1}{2} \int_{z(\delta)}^{\infty} \operatorname{Ai}(x) \mathrm{d} x=\delta
$$




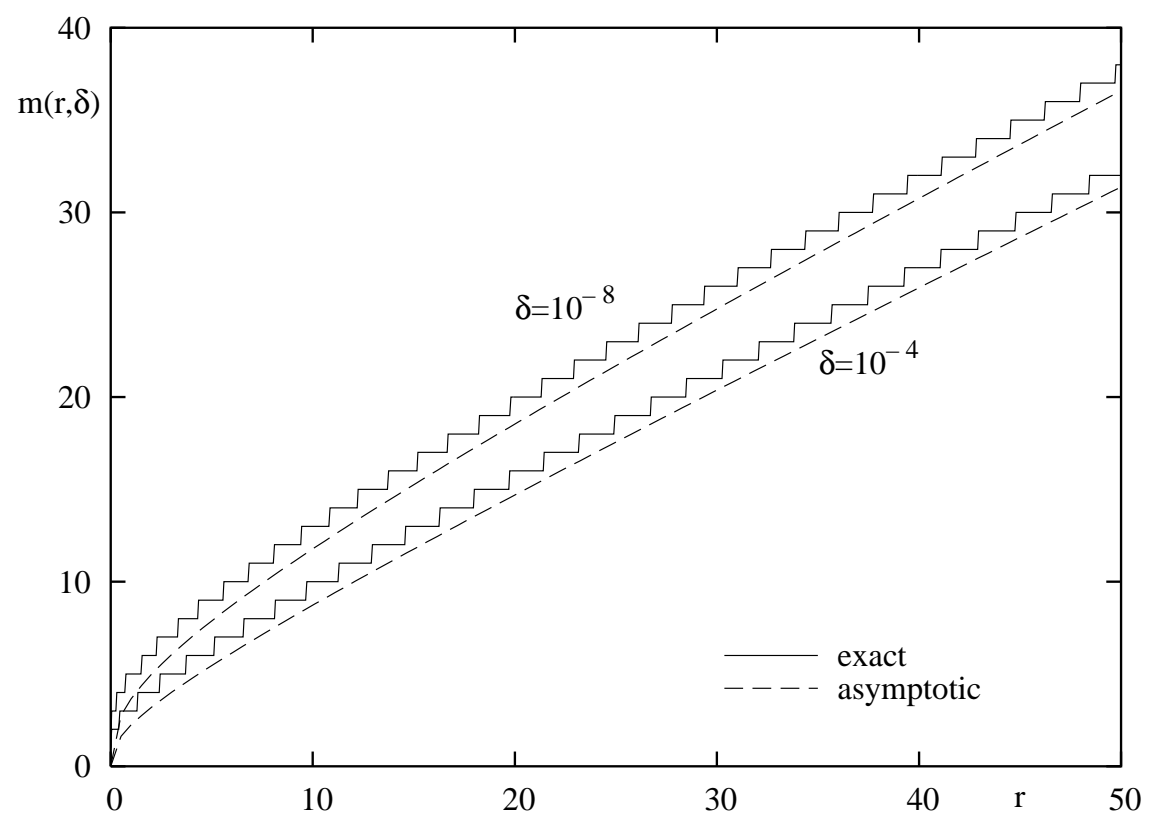

Figure 12: For the bounds $\delta=10^{-4}$ and $\delta=10^{-8}$ of the sum over Bessel functions (87) the result of the exact computation of $m(r, \delta)$ (full curves) and the asymptotic result (96) are compared. The asymptotic results approaches the exact one slowly from below with a rate $O\left(r^{-1 / 3}\right)$.

and then (90) defines together with (94) a function $m(r, \delta)$ such that

$$
\sum_{l=[m(r, \delta)+1]}^{\infty}\left|J_{2 l}(r)\right|=\delta+O\left(r^{-1 / 3}\right) .
$$

By solving (90) for large $r$, we see that we have to take approximately

$$
m(r, \delta) \sim \frac{r}{2}+\frac{z}{2}\left(\frac{r}{2}\right)^{1 / 3}
$$

terms in the sum (28) over $l$ into account such that the error is $\delta+O\left(r^{-1 / 3}\right)$.

For instance, if we require $\delta=10^{-4}$, then (94) gives $z(\delta)=4.359 \ldots$; for $\delta=10^{-8}$ one gets $z(\delta)=7.925 \ldots$ In fig. 12 we show for these choices of $z$ the asymptotic result (96) compared to the exact computation, corresponding to (87). The asymptotic results approaches the exact one slowly from below; in the plotted region a constant offset by two compared to (96) gives a good bound for $m(r, \delta)$.

\section{References}

[1] M. V. Berry: Regular and irregular semiclassical wavefunctions, J. Phys. A 10 (1977) 2083-2091.

[2] S. W. McDonald and A. N. Kaufmann: Wave chaos in the stadium: Statistical properties of short-wave solutions of the Helmholtz equation, Phys. Rev. A 37 (1988) 3067-3086. 
[3] R. Aurich and F. Steiner: Statistical properties of highly excited quantum eigenstates of a strongly chaotic system, Physica D 64 (1993) 185-214.

[4] B. Li and M. Robnik: Statistical properties of high-lying chaotic eigenstates, J. Phys. A 27 (1994) 5509-5523.

[5] D. Robert: Semi-classical approximation in quantum mechanics. A survey of old and recent mathematical results, Helv. Phys. Acta 71 (1998) 1 44-116.

[6] A. I. Shnirelman: Ergodic properties of eigenfunctions (in Russian), Usp. Math. Nauk 29 (1974) 181-182.

[7] S. Zelditch: Uniform distribution of eigenfunctions on compact hyperbolic surfaces, Duke. Math. J. 55 (1987) 919-941.

[8] Y. Colin de Verdière: Ergodicité et fonctions propres du laplacien (in French), Commun. Math. Phys. 102 (1985) 497-502.

[9] B. Helffer, A. Martinez and D. Robert: Ergodicité et limite semi-classique (in French), Commun. Math. Phys. 109 (1987) 313-326.

[10] P. Gérard and E. Leichtnam: Ergodic properties of eigenfunctions for the Dirichlet problem, Duke Math. J. 71 (1993) 559-607.

[11] S. Zelditch and M. Zworski: Ergodicity of eigenfunctions for ergodic billiards, Commun. Math. Phys. 175 (1996) 673-682.

[12] A. Bäcker, R. Schubert and P. Stifter: Rate of quantum ergodicity in Euclidean billiards, Phys. Rev. E 57 (1998) 5425-5447, erratum ibid. 58 (1998) 5192.

[13] A. Voros: Semi-classical approximations, Annales de l'Institut Henri Poincaré A 24 (1976) 31-90.

[14] A. Voros: Asymptotic $\hbar$-expansions of stationary quantum states, Annales de l'Institut Henri Poincaré A 26 (1977) 343-403.

[15] M. V. Berry: Semiclassical mechanics of regular and irregular motion, in: Comportement Chaotique des Systèmes Déterministes - Chaotic Behaviour of Deterministic Systems (Eds. G. Iooss, R. H. G. Hellemann and R. Stora), 171-271, North-Holland, Amsterdam, (1983).

[16] B. Eckhardt, U. Dörr, U. Kuhl and H.-J. Stöckmann: Correlations of electromagnetic fields in chaotic cavities, Europhys. Lett. 46 (1999) 134-140.

[17] M. Srednicki and F. Stiernelof: Gaussian fluctuations in chaotic eigenstates, J. Phys. A 29 (1996) 5817-5826.

[18] M. Srednicki: Gaussian random eigenfunctions and spatial correlations in quantum dots, Phys. Rev. E 54 (1996) 954-955.

[19] S. Hortikar and M. Srednicki: Correlations in chaotic eigenfunctions at large separation, Phys. Rev. Lett. 80 (1998) 1646-1649. 
[20] S. Hortikar and M. Srednicki: Random matrix elements and eigenfunctions in chaotic systems, Phys. Rev. E 57 (1998) 7313-7316.

[21] M. Shapiro and G. Goelman: Onset of chaos in an isolated energy eigenstate, Phys. Rev. Lett. 53 (1984) 1714-1717.

[22] M. Shapiro, J. Ronkin and P. Brumer: Scaling laws and correlation length of quantum and classical ergodic states, Chem. Phys. Lett. 148 (1988) 2,3 177-182.

[23] G. Veble, M. Robnik and J. Liu: Study of regular and irregular states in generic systems, J. Phys. A 32 (1999) 6423-6444.

[24] R. Schubert: Semiclassical localization in phase space, Ph.D. thesis, Abteilung Theoretische Physik, Universität Ulm, (2001).

[25] L. A. Bunimovich: On ergodic properties of certain billiards, Funct. Anal. Appl. 8 (1974) $254-255$.

[26] L. A. Bunimovich: On the ergodic properties of nowhere dispersing billiards, Commun. Math. Phys. 65 (1979) 295-312.

[27] M. Robnik: Classical dynamics of a family of billiards with analytic boundaries, J. Phys. A 16 (1983) 3971-3986.

[28] M. Robnik: Quantising a generic family of billiards with analytic boundaries, J. Phys. A 17 (1984) 1049-1074.

[29] M. Wojtkowski: Principles for the design of billiards with nonvanishing Lyapunov exponents, Commun. Math. Phys. 105 (1986) 391-414.

[30] D. Szász: On the K-property of some planar hyperbolic billiards, Commun. Math. Phys. 145 (1992) 595-604.

[31] R. Markarian: New ergodic billiards: exact results, Nonlinearity 6 (1993) 819-841.

[32] T. Prosen and M. Robnik: private communication.

[33] T. Prosen and M. Robnik: Energy level statistics in the transition region between integrability and chaos, J. Phys. A 26 (1993) 2371-2387.

[34] R. J. Riddel Jr.: Boundary-distribution solution of the Helmholtz equation for a region with corners, J. Comp. Phys. 31 (1979) 21-41.

[35] M. V. Berry and M. Wilkinson: Diabolical points in the spectra of triangles, Proc. R. Soc. London Ser. A 392 (1984) 15-43.

[36] A. Bäcker: Classical and Quantum Chaos in Billiards, Ph.D. thesis, Abteilung Theoretische Physik, Universität Ulm, (1998).

[37] E. Vergini and M. Saraceno: Calculation of highly excited states of billiards, Phys. Rev. E 52 (1995) 2204-2207.

[38] E. J. Heller: Bound-state eigenfunctions of classically chaotic Hamiltonian systems: Scars of periodic orbits, Phys. Rev. Lett. 53 (1984) 1515-1518. 
[39] Y. Y. Bai, G. Hose, K. Stefański and H. S. Taylor: Born-Oppenheimer adiabatic mechanism for regularity of states in the quantum stadium billiard, Phys. Rev. A 31 (1985) 2821-2826.

[40] P. W. O'Connor and E. J. Heller: Quantum localization for a strongly classically chaotic system, Phys. Rev. Lett. 61 (1988) 2288-2291.

[41] G. Tanner: How chaotic is the stadium billiard? A semiclassical analysis, J. Phys. A 30 (1997) 2863-2888.

[42] A. Bäcker, R. Schubert and P. Stifter: On the number of bouncing-ball modes in billiards, J. Phys. A 30 (1997) 6783-6795.

[43] M. Abramowitz and I. A. Stegun (eds.): Pocketbook of Mathematical Functions, Verlag Harri Deutsch, Thun - Frankfurt/Main, abridged edn., (1984).

[44] G. B. Folland: Harmonic Analysis in Phase Space, vol. 122 of Annals of Mathematics Studies, Princeton University Press, Princeton, (1989).

[45] K. Życzkowski: Classical and quantum billiards, integrable, nonintegrable, and pseudointegrable, Acta Physica Polonica B 23 (1992) 245-270.

[46] A. Bäcker and R. Schubert: Chaotic eigenfunctions in momentum space, J. Phys. A 32 (1999) 4795-4815.

[47] J. M. Tualle and A. Voros: Normal modes of billiards portrayed in the stellar (or nodal) representation, Chaos, Solitons and Fractals 5 (1995) 1085-1102.

[48] F. P. Simonotti, E. Vergini and M. Saraceno: Quantitative study of scars in the boundary section of the stadium billiard, Phys. Rev. E 56 (1997) 3859-3867.

[49] A. Bäcker and R. Schubert: Amplitude distribution of eigenfunctions in mixed systems, preprint, 13 pages (2001).

[50] I. C. Percival: Regular and irregular spectra, J. Phys. B 6 (1973) L229-L232.

[51] O. Bohigas, S. Tomsovic and D. Ullmo: Dynamical quasidegeneracies and separation of regular and irregular quantum levels, Phys. Rev. Lett. 64 (1990) 1479-1482.

[52] T. Prosen and M. Robnik: Survey of the eigenfunctions of a billiard system between integrability and chaos, J. Phys. A 26 (1993) 5365-5373.

[53] B. Li and M. Robnik: Separating the regular and irregular energy levels and their statistics in a Hamiltonian system with mixed classical dynamics, J. Phys. A 28 (1995) 4843-4857.

[54] B. Li and M. Robnik: Geometry of high-lying eigenfunctions in a plane billiard system having mixed-type classical dynamics, J. Phys. A 28 (1995) 2799-2818.

[55] G. Carlo, E. Vergini and A. Fendrik: Numerical verification of Percival's conjecture in a quantum billiard, Phys. Rev. E 57 (1998) 5397-5403.

[56] B. Eckhardt, S. Fishman, J. Keating, O. Agam, J. Main and K. Müller: Approach to ergodicity in quantum wave functions, Phys. Rev. E 52 (1995) 5893-5903. 